\title{
A New Generalized Log-logistic and Modified Weibull Distribution with Applications
}

\author{
Broderick O. Oluyede ${ }^{1}$, Huybrechts F. Bindele ${ }^{2}$, Boikanyo Makubate ${ }^{3} \&$ Shujiao Huang $^{4}$ \\ ${ }^{1}$ Department of Mathematical Sciences, Georgia Southern University, USA \\ 2 Department of Mathematics and Statistics, University of South Alabama, USA \\ ${ }^{3}$ Department of Mathematics and Statistical Sciences, Botswana International University of Science and Technology, \\ USA \\ ${ }^{4}$ BBVA Compass, Houston, TX, USA \\ Correspondence: B. O. Oluyede, Department of Mathematical Sciences, Georgia Southern University, Statesboro, GA, \\ 30460, USA.
}

Received: February 15, 2018 Accepted: March 7, 2018 Online Published: April 18, 2018

doi:10.5539/ijsp.v7n3p72 URL: https://doi.org/10.5539/ijsp.v7n3p72

\begin{abstract}
A new generalized distribution called the log-logistic modified Weibull (LLoGMW) distribution is presented. This distribution includes many submodels such as the log-logistic modified Rayleigh, log-logistic modified exponential, log-logistic Weibull, log-logistic Rayleigh, log-logistic exponential, log-logistic, Weibull, Rayleigh and exponential distributions as special cases. Structural properties of the distribution including the hazard function, reverse hazard function, quantile function, probability weighted moments, moments, conditional moments, mean deviations, Bonferroni and Lorenz curves, distribution of order statistics, L-moments and Rényi entropy are derived. Model parameters are estimated based on the method of maximum likelihood. Finally, real data examples are presented to illustrate the usefulness and applicability of the model.
\end{abstract}

Keywords: Generalized Distribution, Log-logistic Distribution, Modified Weibull Distribution, Log-logistic Modified Weibull Distribution, Probability Weighted Moments, L-Moments, Maximum Likelihood Estimation

\section{Introduction}

Distribution theory because of its widespread applications in many fields such as finance, economics, physics, just to cite a few, has spawned the statistical literature. From univariate to multivariate distributions, a lot of important new contributions have been presented. Such contributions include those of Eugene, Famoye \& Lee (2002) dealing with the beta-normal distribution, Oluyede \& Yang (2015) on the beta generalized Lindley distribution, Oluyede, Huang \& Pararai (2014) on the gamma-Dagum distribution, as well general family of univariate distributions generated from Weibull distribution introduced by Gurvich, DiBenedetto \& Ranade (1997). The cumulative distribution function (cdf) given by Gurvich et al. (1997) is

$$
G(x ; \alpha, \boldsymbol{\theta})=1-\exp [-\alpha H(x ; \boldsymbol{\theta}], \quad x \in C, \alpha>0,
$$

where $C$ is a subset of $\mathbb{R}$ and $H(x ; \boldsymbol{\theta})$ is a non-negative monotonically increasing function that depends on the vector of parameters $\boldsymbol{\theta}$. The corresponding probability density function (pdf) is given by

$$
g(x ; \alpha, \boldsymbol{\theta})=\alpha \exp [-\alpha H(x ; \boldsymbol{\theta})] h(x ; \boldsymbol{\theta}),
$$

where $h(x ; \boldsymbol{\theta})$ is the derivative of $H(x ; \boldsymbol{\theta})$. Different choices of the function $H(x ; \boldsymbol{\theta})$ lead to different models. Such choices are for example, $H(x ; \boldsymbol{\theta})=x$, for the exponential distribution, $H(x ; \boldsymbol{\theta})=x^{2}$, for the Rayleigh distribution, $H(x ; \boldsymbol{\theta})=$ $\log (x / k)$, for the Pareto distribution, and $H(x ; \boldsymbol{\theta})=(\exp (\beta x)-1) / \beta$, for the Gompertz distribution.

Several useful ways for generating new probability distributions from classic ones to relative new distributions have been proposed in the literature. Nelson (1982) stated that distributions with bathtub-shaped failure rate are often complex and difficult to model. One example of such a distribution was proposed by Hjorth (1980). To lessen the complexity of these distribution, while Rajarshi and Rajarshi (1988) presented a revised version of these distributions, Haupt and Schabe (1992) on the other hand put forward a new lifetime model with bathtub-shaped failure rates. These models are unfortunately not sufficient enough to address various practical situations. Then, new classes of distributions based on modified versions of the Weibull distribution were presented to satisfy non-monotonic failure rate. For such distributions, the reader can refer to Mudholkar and Srivastava (1993), and Pham and Lai (2007) for more details. These authors in their papers, summarized some generalizations of Weibull distribution. Additional generalized distributions include the 
exponentiated Weibull (EW) (Gupta and Kundu (2001)), the modified Weibull (MW) (Lai, Xie \& Murthy (2003)) and the beta exponential (BE) (Nadarajah \& Kotz (2005)). Recent extensions are the generalized modified Weibull (GMW) (Carrasco, Ortega \& Cordeiro (2008)), the beta modified Weibull (BMW) (Nadarajah Cordeiro \& Ortega (2011)), (Siva, Ortega \& Cordeiro (2010)), the Weibull-G family (Bourguignon, Silva \& Cordeiro (2014)), the gamma-exponentiated Weibull (GEW) (Pinho, Cordeiro \& Nobre (2012)), gamma Weibull-G family (Oluyede, Pu, Makubate \& Qui (2018)) and the gamma generalized modified Weibull (GGMW) (Oluyede, Huang \& Yang (2015)). A new statistical distribution for characterizing the random strength of brittle materials was developed in Gurvich et al. (1997).

In this paper, we propose and study a new generalized distribution called the log-logistic modified Weibull distribution. The primary motivations for considering this new distribution are the advantages it offers with respect to having hazard functions that exhibits increasing, decreasing and bathtub shapes, as well as the versatility and flexibility in modeling lifetime data. This new distribution inherits these desirable properties and also has quite a variety of shapes.

There is another added advantage to this model, in that it also has added dispersion parameter, depending on the overall form that accounts for the scale of the underlying random variable. This distribution also has exponential dumping in the upper tail making the distribution suitable for modeling samples that display power behavior for intermediate observations and decrease in tail probability for large observations or beyond a certain threshold or specified value. Our proposed new distribution also generalizes the log-logistic and modified Weibull distributions. Some structural properties of this distribution are obtained and based on the method of maximum likelihood, estimates of the model parameters are presented.

To this end, the remainder of the paper is organized as follows. Section 2 is concerned with the presentation of the generalized distribution, log-logistic modified Weibull distribution as well as it's hazard and reverse hazard functions, quantile function, and various sub-models. In section 3, probability weighted moments, moments, conditional moments and moment generating function are presented. Mean deviations, Bonferroni and Lorenz curves are derived in section 4. Section 5 is concerned with Rényi entropy, distribution of order statistics and L-moments. Estimates of model parameters and comparisons with other models are presented in section 6. A Monte Carlo simulation study is conducted to examine the bias and mean square error of the maximum likelihood estimates in section 7. Estimation with censored samples are given in section 8. Applications of the proposed model to a real data are given in section 9. The paper end with concluding remarks in section 10 .

\section{Generalized Extended Weibull Distribution}

The generalized class of distributions referred to as the generalized extended Weibull (GEW) distribution is given by

$$
G(x ; \boldsymbol{\theta}, \boldsymbol{\xi})=1-B(x ; \boldsymbol{\xi}) \exp (-\alpha H(x ; \boldsymbol{\theta})),
$$

where $B(x ; \boldsymbol{\xi})>0$ is a continuous function, $H(x ; \boldsymbol{\theta})$ is a non-negative monotonically increasing function that depends on the vector of parameters $\boldsymbol{\theta}$, and $\boldsymbol{\xi}$ is a vector of parameters corresponding to the function $B(x ; \boldsymbol{\xi})$. Putting $H(x ; \boldsymbol{\theta})=x^{\beta} e^{\lambda x}$, the generalized extended modified Weibull distribution is obtained. For $B(x ; \xi)=\left(1+\left(\frac{x}{s}\right)^{c}\right)^{-1}$ and $H(x ; \boldsymbol{\theta})=x^{\beta} e^{\lambda x}$, we get the $\log \operatorname{logistic}$ modified Weibull distribution. The choice of the functions $B(x ; \boldsymbol{\xi})$ and $H(x ; \boldsymbol{\theta})$ including in particular, $H(x ; \boldsymbol{\theta})=x^{\beta} e^{\lambda x}$, defines a large class of distributions with applications in several areas including reliability, biometry, economics, finance, medicine and survival analysis to mention just a few areas. To avoid potential problems or issues with over parametrization, the parameter vectors $\boldsymbol{\xi}$ and $\boldsymbol{\theta}$ can be restricted to two component vectors, respectively.

We can also have the following motivation for the model under study. Consider a series system and assume that the lifetime of the components follow the log-logistic and modified Weibull distributions with reliability functions $R_{1}(t)=\left(1+\left(\frac{t}{s}\right)^{c}\right)^{-1}$ and $R_{2}(t)=e^{-\alpha t^{\beta} e^{\lambda t}}$, respectively. The reliability $R(t)=P(T>t)$ of the system is given by $R(t)=\prod_{i=1}^{2} R_{i}(t)$. This leads to the log-logistic modified Weibull reliability function. Note that in some context, a series model is referred to as a competing risk model.

\subsection{The Log-Logistic Modified Weibull Distribution}

Some statistical properties of the new log-logistic modified Weibull (LLoGMW) distribution, including pdf, cdf, hazard and reverse hazard functions are presented. Plots of the hazard rate function for selected values of the model parameters are also given. First of all, we present the log-logistic and modified Weibull distributions. The log-logistic distribution attract special attention because it has varying degrees of skewness and kurtosis. Furthermore, this distribution have applications in a wide variety of areas in statistics and applied mathematics including modeling events associated with fracture roughness, life testing, operational risk, option market price distributions, forestry, meteorology, modeling crop prices, software reliability growth, and reliability analysis. The cdf of the log-logistic distribution is given by

$$
F_{L L O G}(x)=1-\left(1+\left(\frac{x}{s}\right)^{c}\right)^{-1}, \quad \text { for } s, c, \text { and } x \geq 0 .
$$


The parameters $c$ and $s$ are shape and scale parameters, respectively. The log-logistic distribution is a special case of the Burr XII distribution. See Burr (1942), (1973) for additional details.

The modified Weibull (MW) (Lai et al. (2003)) distribution is given by

$$
F_{M W}(x ; \alpha, \beta, \lambda)=1-\exp \left(-\alpha x^{\beta} e^{\lambda x}\right), \quad x \geq 0, \alpha>0, \beta>0, \lambda \geq 0 .
$$

The corresponding pdf is given by

$$
f_{M W}(x ; \alpha, \beta, \lambda)=\alpha x^{\beta-1} e^{\lambda x}(\beta+\lambda x) \exp \left(-\alpha x^{\beta} e^{\lambda x}\right),
$$

for $x \geq 0, \alpha>0, \beta>0$, and $\lambda \geq 0$. It is noted that $\alpha$ controls the scale of the distribution, $\beta$ controls the shape, whereas $\lambda$ can be considered to be an accelerating factor in the imperfection time and a factor of fragility in the survival of the individual as time increases. The Weibull distribution is obtained by setting $\lambda=0$. This distribution is well known and has been extensively used for modeling data in several areas including reliability.

Now, consider the log-logistic modified Weibull (LLoGMW) distribution obtained by setting $H(x ; \boldsymbol{\theta})=x^{\beta} e^{\lambda x}$ and $B(x ; \boldsymbol{\xi})=$ $\left(1+\left(\frac{x}{s}\right)^{c}\right)^{-1}$, (or via the competing risk model), to obtain

$$
G_{L L O G M W}(x)=1-\left(1+\left(\frac{x}{s}\right)^{c}\right)^{-1} \exp \left(-\alpha x^{\beta} e^{\lambda x}\right),
$$

for $s, c, \alpha, \beta>0, \lambda \geq 0$ and $x \geq 0$. The corresponding LLoGMW pdf is given by

$$
g_{\text {LLoGMW }}(x)=e^{-\alpha x^{\beta} e^{\lambda x}}\left[1+\left(\frac{x}{s}\right)^{c}\right]^{-1}\left\{\alpha x^{\beta-1} e^{\lambda x}(\beta+\lambda x)+\frac{c x^{c-1}}{\left(s^{c}+x^{c}\right)}\right\},
$$

$s, c, \alpha, \beta>0, \lambda \geq 0$ and $x \geq 0$. For simplicity, we suppress the index-name on $G_{L L O G M W}$ and $g_{L L O G M W}$ so that $G$ and $g$ now stand for the cdf and pdf of the LLoGMW, respectively.

As shown in the plot below, depending on the values of the parameters, the density and hazard functions can exhibit different behavior. Figure 1 displays graphs of the pdf for selected values of the model parameters. The plot suggests that the LLoGMW pdf can be right skewed or decreasing for the selected values of the parameters.

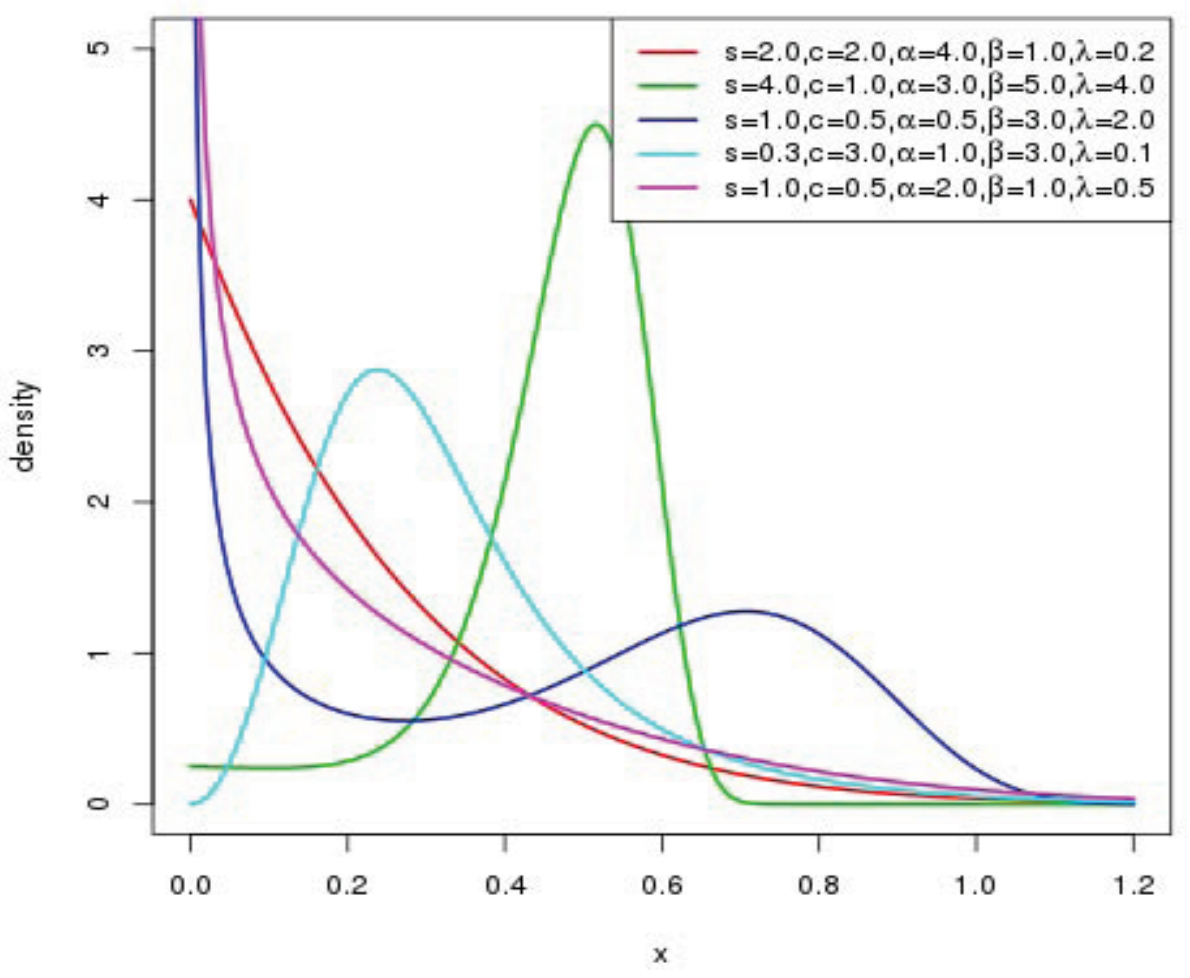

Figure 1. PDF Plots of the LLoGMW Distribution 


\subsection{Some New and Known Sub-models}

Under the considered choice of the functions $B(x ; \boldsymbol{\xi})$ and $H(x ; \boldsymbol{\theta})$, several new and well known distributions can be obtained. For the LLoGMW distribution, the sub-models include the following distributions:

- If $\beta=1$, we obtain the log-logistic modified exponential (LLoGME) distribution.

- If $\beta=2$, we obtain the log-logistic modified Rayleigh (LLoGMR) distribution.

- If $\lambda=0$, we obtain the log-logistic Weibull (LLoGW) distribution.

- If $\lambda=0$, and $\beta=1$, we obtain the log-logistic exponential (LLoGE) distribution.

- When $\alpha \rightarrow 0^{+}$, we have the log-logistic (LLoG) distribution.

- If $\lambda=0$, and $\beta=2$, we have the log-logistic Rayleigh (LLoGR) distribution.

- If $c=1, s \rightarrow \infty$, and $\lambda=0$, then we obtain Weibull (W) distribution.

- If $c=1, s \rightarrow \infty, \lambda=0$, and $\beta=1$, then we have the exponential (E) distribution.

- If $c=1, s \rightarrow \infty, \lambda=0$, and $\beta=2$, then we have Rayleigh (R) distribution.

- If $c=1$, then the LLoGMW cdf reduces to the four parameter distribution with cdf given by

$$
G(x)=1-\left(1+\left(\frac{x}{s}\right)\right)^{-1} \exp \left(-\alpha x^{\beta} e^{\lambda x}\right),
$$

for $s, \alpha, \beta>0, \lambda \geq 0$, and $x \geq 0$.

- If $c=\beta=1$ and $\lambda=0$, then the LLoGMW cdf reduces to the two parameter distribution given by

$$
G(x)=1-\left(1+\left(\frac{x}{s}\right)\right)^{-1} \exp (-\alpha x)
$$

for $s, \alpha>0$, and $x \geq 0$.

- If $c=1, \lambda=0$ and $\beta=2$, then the LLoGMW cdf reduces to the two parameter model with the cdf

$$
G(x)=1-\left(1+\left(\frac{x}{s}\right)\right)^{-1} \exp \left(-\alpha x^{2}\right),
$$

for $s, \alpha>0$, and $x \geq 0$.

\subsection{Hazard and Reverse Hazard Functions}

We present the hazard and reverse hazard functions of the LLoGMW distribution. The hazard and reverse hazard functions of the LLoGMW distribution are respectively given by

$$
\begin{aligned}
h_{G}(x) & =\left\{\alpha x^{\beta-1} e^{\lambda x}(\beta+\lambda x)+\left(1+\left(\frac{x}{s}\right)^{c}\right)^{-1} \frac{c}{s}\left(\frac{x}{s}\right)^{c-1}\right\} \\
& =h_{F_{M W}}(x)+h_{F_{B}}(x),
\end{aligned}
$$

and

$$
\tau_{G}(x)=\left[1-\left(1+\left(\frac{x}{s}\right)^{c}\right)^{-1} e^{-\alpha x^{\beta} e^{\lambda x}}\right]^{-1} e^{-\alpha x^{\beta} e^{\lambda x}}\left[1+\left(\frac{x}{s}\right)^{c}\right]^{-1}\left\{\alpha x^{\beta-1} e^{\lambda x}(\beta+\lambda x)+\frac{c x^{c-1}}{\left(s^{c}+x^{c}\right)}\right\}
$$

for $x \geq 0, s, c, \alpha, \beta>0, \lambda \geq 0$, where $h_{F_{M W}}(x)$ and $h_{F_{B}}(x)$ are the hazard functions of the modified Weibull and Burr XII distributions. The limiting behavior of the hazard function of the LLoGMW distribution can be readily established and is as follows: 
- Note that,

$$
\lim _{x \rightarrow 0} h_{G}(x)= \begin{cases}\alpha+\frac{1}{s}, & c=1, \beta=1, \\ \frac{1}{s}, & c=1, \beta>1, \\ \infty, & 0<c \leq 1,0<\beta \leq 1, \\ \alpha, & c>1, \beta=1, \\ 0, & c>1, \beta>1 .\end{cases}
$$

- For $\beta>0$, and for each $c>0, \lim _{x \rightarrow \infty} h_{G}(x)=\infty$.

Figure 2 displays graphs of the hazard function for different choice of model parameters. The graphs exhibit increasing, bathtub and upside down bathtub followed by bathtub shapes for the selected values of the model parameters. This very attractive flexibility makes the LLoGMW hazard function useful and suitable for non-monotonic empirical hazard behaviors which are far more likely to be encountered in practice or real life situations.

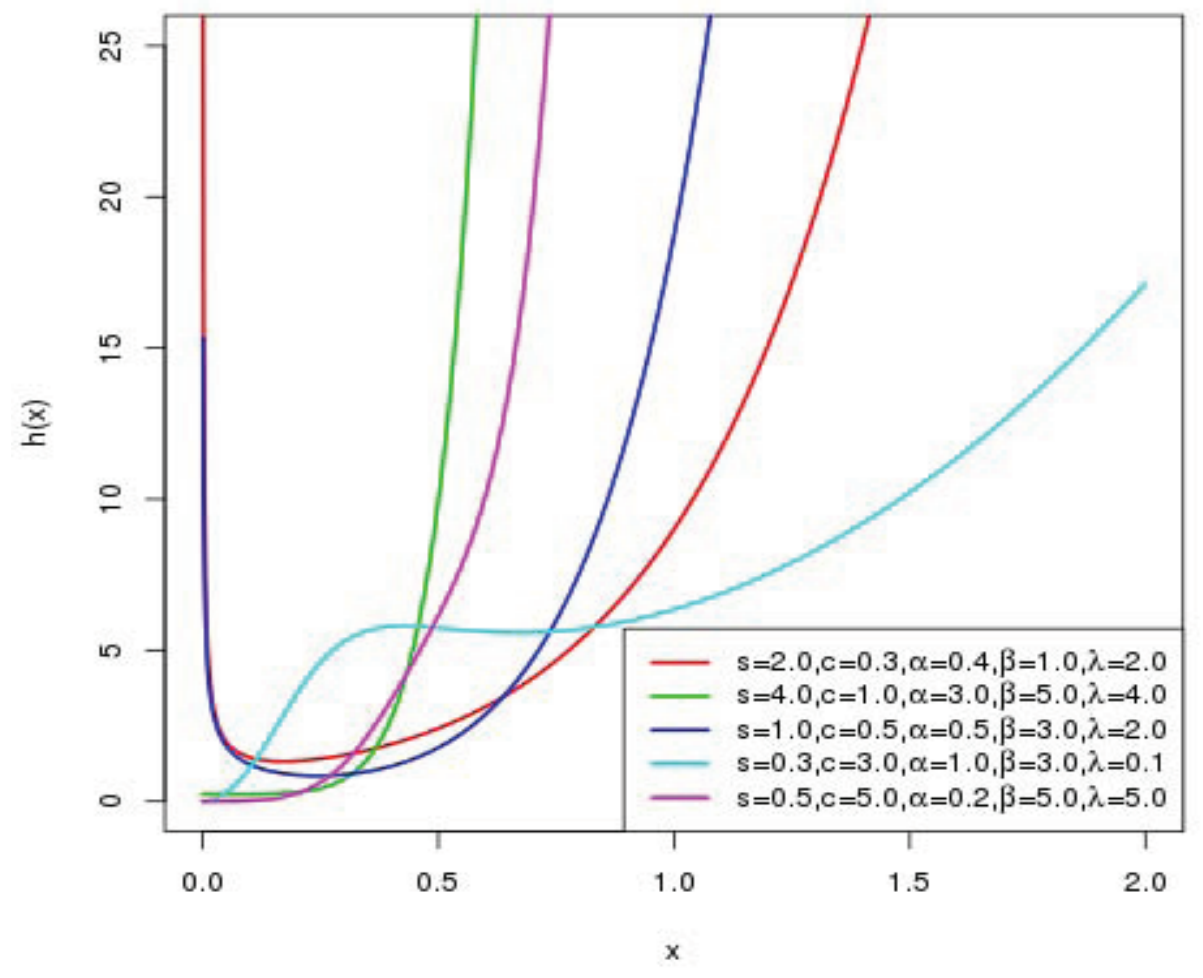

Figure 2. Hazard Function Plots of the LLoGMW Distribution

\subsection{Quantile Function}

The quantile function of the LLoGMW distribution is obtained by inverting $G(x)=u$, where $0 \leq u \leq 1$, and

$$
G(x)=1-\left(1+\left(\frac{x}{s}\right)^{c}\right)^{-1} e^{-\alpha x^{\beta} e^{\lambda x}} .
$$

This is equivalent to solving the non-linear equation

$$
\alpha x^{\beta} e^{\lambda x}+\log \left(1+\left(\frac{x}{s}\right)^{c}\right)+\log (1-u)=0,
$$

which can be done using numerical methods. Consequently, random number can be generated based on equation (14). Some quantile results for selected parameters are given in Table 1. 
Table 1. Table of Quantiles

\begin{tabular}{c|ccccc}
\hline & \multicolumn{5}{|c}{$(s, c, \alpha, \beta, \lambda)$} \\
\hline $\mathrm{u}$ & $(2,0.3,0.4,1,2)$ & $(4,1,3,5,4)$ & $(1,0.5,0.5,3,0.1)$ & $(0.5,5,0.2,5,5)$ & $(0.5,2,2,0.5,1)$ \\
\hline 0.1 & 0.001294753 & 0.3112413 & 0.01232197 & 0.3200301 & 0.002746775 \\
0.2 & 0.017397929 & 0.3905252 & 0.06242341 & 0.3752197 & 0.012095361 \\
0.3 & 0.077807571 & 0.4320222 & 0.18008480 & 0.4163027 & 0.029402413 \\
0.4 & 0.188905028 & 0.4621197 & 0.38289709 & 0.4524904 & 0.055597583 \\
0.5 & 0.316724995 & 0.4872344 & 0.60513838 & 0.4871860 & 0.090950913 \\
0.6 & 0.440165155 & 0.5100256 & 0.80003516 & 0.5226001 & 0.135980227 \\
0.7 & 0.558978966 & 0.5323736 & 0.97830995 & 0.5608002 & 0.192883778 \\
0.8 & 0.681372195 & 0.5563050 & 1.16080064 & 0.6046572 & 0.268591470 \\
0.9 & 0.827661482 & 0.5861878 & 1.38472324 & 0.6608587 & 0.387162609 \\
\hline
\end{tabular}

\section{Probability Weighted Moments, Moments, Conditional Moments and Moment Generating Function}

We now derive probability weighted moments (PWMs) (Greenwood, Landwehr, Matalas, and Willis (1997)), moments, moment generating function and conditional moments for the LLoGMW distribution. We also present the PWMs for the special case when $\lambda=0$, that is, the PWMs for the log-logistic Weibull distribution. The PWMs of the submodels given in section 2 can be readily obtained from those of the generalized distribution. Moments are necessary and crucial in any statistical analysis, especially in applications. Moments can be used to study the most important features and characteristics of a distribution (e.g., tendency, dispersion, skewness and kurtosis). The probability weighted moments (PWMs) of the LLoGMW distribution is given by

$$
\begin{aligned}
E\left[X^{r} G^{l}(X) \bar{G}^{m}(X)\right] & =\int_{0}^{\infty} x^{r} G^{l}(x) \bar{G}^{m}(x) g(x) d x \\
& =\int_{0}^{\infty} x^{r}(1-\bar{G}(x))^{l} \bar{G}^{m}(x) g(x) d x \\
& =\sum_{j=0}^{\infty} \frac{(-1)^{j} \Gamma(l+1) E\left(X^{r}(\bar{G}(X))^{j+m}\right)}{\Gamma(l+1-j) \Gamma(j+1)},
\end{aligned}
$$

where we have used the binomial expansion $(1-\bar{G}(x))^{l}=\sum_{j=0}^{\infty} \frac{(-1)^{j} \Gamma(l+1)[\bar{G}(x)]^{j}}{\Gamma(l+1-j) \Gamma(j+1)}$. Note that

$$
\begin{aligned}
& E\left[X^{r}(\bar{G}(X))^{j+m}\right]=\int_{0}^{\infty} x^{r}\left(1+\left(\frac{x}{s}\right)^{c}\right)^{-(m+j)} e^{-(m+j) \alpha x^{\beta} e^{\lambda x}}\left(1+\left(\frac{x}{s}\right)^{c}\right)^{-1} \\
& \times e^{-\alpha x^{\beta} e^{\lambda x}}\left[\frac{c}{s}\left(\frac{x}{s}\right)^{c-1}\left(1+\left(\frac{x}{s}\right)^{c}\right)^{-1}+\alpha x^{\beta-1} e^{\lambda x}(\beta+\lambda x)\right] d x \\
& =\sum_{k=0}^{\infty} \frac{(-1)^{k}(m+j+1)^{k}}{k !} \int_{0}^{\infty} x^{r}\left(1+\left(\frac{x}{s}\right)^{c}\right)^{-(m+j+1)}\left(\alpha x^{\beta} e^{\lambda x}\right)^{k} \\
& \times\left[\frac{c}{s}\left(\frac{x}{s}\right)^{c-1}\left(1+\left(\frac{x}{s}\right)^{c}\right)^{-1}+\alpha x^{\beta-1} e^{\lambda x}(\beta+\lambda x)\right] d x \\
& =\sum_{k=0}^{\infty} \frac{(-1)^{k}(m+j+1)^{k} \alpha^{k}}{k !}\left[\frac{c}{s^{c}} \int_{0}^{\infty} x^{r+k \beta+c-1}\left(1+\left(\frac{x}{s}\right)^{c}\right)^{-(m+j+2)} \sum_{p=0}^{\infty} \frac{(\lambda k x)^{p}}{p !} d x\right. \\
& \left.+\alpha \int_{0}^{\infty} x^{r+(k+1) \beta-1}\left(1+\left(\frac{x}{s}\right)^{c}\right)^{-(m+j+1)} \times \sum_{p=0}^{\infty} \frac{(\lambda(k+1) x)^{p}}{p !}(\beta+\lambda x) d x\right] .
\end{aligned}
$$


Now, by setting $y=\left(1+(x / s)^{c}\right)^{-1}$, then $x=s((1-y) / y)^{1 / c}$, and $d x=-\frac{s}{c} y^{-2}\left(\frac{1-y}{y}\right)^{(1-c) / c} d y$, so that

$$
\begin{aligned}
& E\left(X^{r}(\bar{G}(X))^{j+m}\right)=\sum_{k=0}^{\infty} \frac{(-1)^{k}((m+j+1) \alpha)^{k}}{k !}\left[\sum_{p=0}^{\infty} \frac{(k \lambda)^{p}}{p !} \frac{c s^{r+k \beta+c+p-1}}{s^{c}} \frac{s}{c}\right. \\
& \times \quad \int_{0}^{1} y^{m+j-\frac{(r+k \beta+p)}{c}}(1-y)^{\frac{r+k \beta+p}{c}} d y+\alpha \sum_{p=0}^{\infty} \frac{(\lambda(k+1))^{p}}{p !}\left(\beta s^{r+\beta(k+1)+p}\left(\frac{s}{c}\right)\right. \\
& \left.\left.\times \quad \int_{0}^{1} y^{m+j-\frac{(r+\beta(k+1)+p+1)}{c}}(1-y)^{\frac{r+\beta(k+1)+p+1}{c}-1} d y+\lambda s^{r+\beta(k+1)+p}\left(\frac{s}{c}\right) \int_{0}^{1} y^{m+j-\frac{(r+\beta(k+1)+p+2)}{c}}(1-y)^{\frac{r+\beta(k+1)+p+2}{c}-1} d y\right)\right] \\
& =\sum_{k=0}^{\infty} \frac{(-1)^{k}(\alpha(m+j+1))^{k}}{k !}\left[\sum_{p=0}^{\infty} \frac{(k \lambda)^{p}}{p !} s^{r+k \beta+p} \mathscr{B}\left(m+j+1-\frac{(r+k \beta+p)}{c}, \frac{r+k \beta+p+c}{c}\right)\right. \\
& +\alpha \sum_{p=0}^{\infty} \frac{(\lambda(k+1))^{p}}{p !}\left(\frac{\beta s^{r+\beta(k+1)+p+1}}{c} \mathscr{B}\left(m+j+1-\frac{(r+\beta(k+1)+p+1)}{c}, \frac{r+\beta(k+1)+p+1}{c}\right)\right. \\
& \left.\left.+\frac{\lambda s^{r+\beta(k+1)+p+1}}{c} \mathscr{B}\left(m+j+1-\frac{(r+\beta(k+1)+p+2)}{c}, \frac{r+\beta(k+1)+p+2}{c}\right)\right)\right],
\end{aligned}
$$

where $\mathscr{B}(a, b)=\int_{0}^{1} t^{a-1}(1-t)^{b-1} d t$ is the beta function.

Consequently, the PWMs of the LLoGMW distribution $E\left[X^{r} G^{l}(X) \bar{G}^{m}(X)\right]$ is given by

$$
\begin{aligned}
E\left[X^{r} G^{l}(X) \bar{G}^{m}(X)\right] & =\sum_{j, k=0}^{\infty} \frac{(-1)^{j+k} \Gamma(l+1)[\alpha(j+m+1)]^{k}}{\Gamma(l+1-j) \Gamma(j+1) k !}\left[\sum_{p=0}^{\infty} \frac{(k \lambda)^{p}}{p !} \mathscr{B}\left(j+m+1-\frac{(r+k \beta+p)}{c}, \frac{r+k \beta+p+c}{c}\right)\right. \\
& +\frac{\alpha}{c} \sum_{p=0}^{\infty} \frac{(\lambda(k+1))^{p}}{p !}\left(\beta s^{r+\beta(k+1)+p+1} \mathscr{B}\left(m+j+1-\frac{(r+\beta(k+1)+p+1)}{c}, \frac{r+\beta(k+1)+p+1}{c}\right)\right. \\
& \left.\left.+\lambda s^{r+\beta(k+1)+p+2} \mathscr{B}\left(m+j+1-\frac{(r+\beta(k+1)+p+2)}{c}, \frac{r+\beta(k+1)+p+2}{c}\right)\right)\right]
\end{aligned}
$$

\section{Special Cases: For $\lambda=0$.}

- When $l=m=0$, we obtain the $r^{\text {th }}$ non-central moments given by

$$
E\left(X^{r}\right)=\sum_{k=0}^{\infty} \frac{(-1)^{k} \alpha^{k} s^{k \beta+r}}{k !}\left(\frac{\alpha \beta s^{\beta}}{c} \mathscr{B}\left(1-\frac{k \beta+\beta+r}{c}, \frac{k \beta+\beta+r}{c}\right)+\mathscr{B}\left(1-\frac{k \beta+r}{c}, 1+\frac{k \beta+r}{c}\right)\right) .
$$

- When $r=l=0$, we have

$$
E\left[\bar{G}^{m}(X)\right]=\sum_{k=0}^{\infty} \frac{(-1)^{k}[\alpha(m+1)]^{k} s^{k \beta}}{k !}\left[\mathscr{B}\left(m+1-\left(\frac{k \beta}{c}\right), \frac{k \beta+c}{c}\right)+\frac{\alpha \beta s^{\beta}}{c} \mathscr{B}\left(m+1-\left(\frac{k \beta+\beta}{c}\right), \frac{k \beta+\beta}{c}\right)\right] .
$$

- When $l=0$, we have, the LLOGMW PWMs reduces to

$$
\begin{aligned}
E\left[X^{r} \bar{G}^{m}(X)\right] & =\sum_{k=0}^{\infty} \frac{(-1)^{k}[\alpha(m+1)]^{k} s^{r+k \beta}}{k !}\left[\mathscr{B}\left(m+1-\left(\frac{r+k \beta}{c}\right), \frac{r+k \beta+c}{c}\right)\right. \\
& \left.+\frac{\alpha \beta s^{\beta}}{c} \mathscr{B}\left(m+1-\left(\frac{r+k \beta+\beta}{c}\right), \frac{r+k \beta+\beta}{c}\right)\right] .
\end{aligned}
$$

- When $m=0$, the LLOGMW PWMs reduces to

$$
\begin{aligned}
E\left[X^{r} G^{l}(X)\right] & =\sum_{j, k=0}^{\infty} \frac{(-1)^{j+k} \Gamma(l+1)[\alpha(j+1)]^{k} s^{r+k \beta}}{\Gamma(l+1-j) \Gamma(j+1) k !} \\
& \times\left[\mathscr{B}\left(j+1-\left(\frac{r+k \beta}{c}\right), \frac{r+k \beta+c}{c}\right)+\frac{\alpha \beta s^{\beta}}{c} \mathscr{B}\left(j+1-\left(\frac{r+k \beta+\beta}{c}\right), \frac{r+k \beta+\beta}{c}\right)\right] .
\end{aligned}
$$

- When $r=m=0$, we have

$$
E\left[G^{l}(X)\right]=\sum_{j, k=0}^{\infty} \frac{(-1)^{j+k} \Gamma(l+1)[\alpha(j+1)]^{k} s^{k \beta}}{\Gamma(l+1-j) \Gamma(j+1) k !}\left[\mathscr{B}\left(j+1-\left(\frac{k \beta}{c}\right), \frac{k \beta+c}{c}\right)+\frac{\alpha \beta s^{\beta}}{c} \mathscr{B}\left(j+1-\left(\frac{k \beta+\beta}{c}\right), \frac{k \beta+\beta}{c}\right)\right] .
$$


Table 2. Table of Moments

\begin{tabular}{c|ccccc}
\hline & \multicolumn{5}{|c}{$(s, c, \alpha, \beta, \lambda)$} \\
\hline & $(2,2,3,0.5,2)$ & $(4,3,5,4,0.1)$ & $(1,0.5,0.5,2,2)$ & $(0.3,3,1,3,3)$ & $(0.5,2,2,1,1)$ \\
\hline$E(X)$ & 0.0845717313 & 0.5957667 & 0.40712891 & 0.306747950 & 0.24692714 \\
$E\left(X^{2}\right)$ & 0.0172422199 & 0.3822143 & 0.25555966 & 0.113004619 & 0.09264615 \\
$E\left(X^{3}\right)$ & 0.0048313719 & 0.2597219 & 0.18148193 & 0.047702328 & 0.04410431 \\
$E\left(X^{4}\right)$ & 0.0016290716 & 0.1849511 & 0.13856127 & 0.022324986 & 0.02478445 \\
$E\left(X^{5}\right)$ & 0.0006243745 & 0.1370070 & 0.11147059 & 0.011309950 & 0.01579174 \\
$E\left(X^{6}\right)$ & 0.0002636515 & 0.1050065 & 0.09345177 & 0.006095448 & 0.01111651 \\
$\mathrm{SD}$ & 0.1004482064 & 0.1651554 & 0.29967601 & 0.137514777 & 0.17796948 \\
$\mathrm{CV}$ & 1.1877279192 & 0.2772149 & 0.73607156 & 0.448298926 & 0.72073682 \\
$\mathrm{CS}$ & 1.6443274503 & -0.1086273 & 0.16019247 & 0.552594423 & 0.99089869 \\
$\mathrm{CK}$ & 5.7084607783 & 2.7524812 & 1.82914011 & 2.885772519 & 3.95001299 \\
\hline
\end{tabular}

- When $r=0$, we have

$$
\begin{aligned}
E\left[G^{l}(X) \bar{G}^{m}(X)\right) & =\sum_{j, k=0}^{\infty} \frac{(-1)^{j+k} \Gamma(l+1)[\alpha(j+m+1)]^{k} s^{k \beta}}{\Gamma(l+1-j) \Gamma(j+1) k !} \\
& \times\left[\mathscr{B}\left(j+m+1-\left(\frac{k \beta}{c}\right), \frac{k \beta+c}{c}\right)+\frac{\alpha \beta s^{\beta}}{c} \mathscr{B}\left(j+m+1-\left(\frac{k \beta+\beta}{c}\right), \frac{k \beta+\beta}{c}\right)\right] .
\end{aligned}
$$

Recall that from the Taylor's series expansion, the function $e^{t x}=\sum_{j=0}^{\infty}(1 / j !)(t x)^{j}$. Thus, the moment generating function (MGF) of the LLoGMW distribution is given by $M_{X}(t)=E\left(e^{t X}\right)=\sum_{n=0}^{\infty}\left(t^{n} / n !\right) E\left(X^{n}\right)$, where $E\left(X^{n}\right)$ is the $n^{\text {th }}$ raw moment of the LLoGMW distribution and is given above. Some moments and related measures including standard deviation (SD), coefficients of variation (CV), coefficients of skewness (CS) and coefficients of kurtosis (CK) for selected values of the model parameters are given in Table 2, where $S D=\left(\mu_{2}-\mu_{1}^{2}\right)^{1 / 2}, C V=\left(\mu_{2} / \mu_{1}^{2}-1\right)^{1 / 2}, C S=\left(\mu_{3}-3 \mu_{1} \mu_{2}+2 \mu_{1}^{3}\right) /\left(\mu_{2}-\mu_{1}^{2}\right)^{3 / 2}$, and $C K=\left(\mu_{4}-4 \mu_{1} \mu_{3}+6 \mu_{1}^{2} \mu_{2}-3 \mu_{1}^{4}\right) /\left(\mu_{2}-\mu_{1}^{2}\right)^{2}$.

\subsection{Conditional Moments}

When it comes to lifetime models, it is often of interest to find the conditional moments and the mean residual lifetime function. The $r^{\text {th }}$ conditional moments for LLoGMW distribution is given by

$$
\begin{aligned}
E\left(X^{r} \mid X>t\right) & =\frac{1}{\bar{G}(t)} \int_{t}^{\infty} x^{r} g(x) d x=\frac{1}{\bar{G}(t)} \int_{t}^{\infty} x^{r} \sum_{k=0}^{\infty} \frac{(-1)^{k} \alpha^{k} x^{\beta k} e^{k \lambda x}}{k !}\left[1+\left(\frac{x}{s}\right)^{c}\right]^{-1} \\
& \times\left[\frac{c}{s}\left(\frac{x}{s}\right)^{c-1}\left(1+\left(\frac{x}{s}\right)^{c}\right)+\alpha x^{\beta-1} e^{\lambda x}(\beta+\lambda x)\right] d x \\
& =\frac{1}{\bar{G}(t)}\left[\sum_{k, m=0}^{\infty} \frac{c}{s^{c}} \frac{(-1)^{k}(k \lambda)^{m} \alpha^{k}}{k ! m !} \int_{t}^{\infty} x^{r+k \beta+m+c-1}\left[1+\left(\frac{x}{s}\right)^{c}\right]^{-2} d x\right. \\
& \left.+\sum_{k, m=0}^{\infty} \frac{c}{s^{c}} \frac{(-1)^{k}((k+1) \lambda)^{m} \alpha^{k}}{k ! m !} \int_{t}^{\infty} x^{r+k \beta+m+c-1}(\beta+\lambda x)\left[1+\left(\frac{x}{s}\right)^{c}\right]^{-1} d x\right]
\end{aligned}
$$

By setting $y=\left(1+(x / s)^{c}\right)^{-1}$, we have

$$
\begin{aligned}
E\left(X^{r} \mid X>t\right) & =\frac{1}{\bar{G}(t)}\left[\sum_{k, m=0}^{\infty} \frac{(-1)^{k+1}(k \lambda)^{m} \alpha^{k}}{k ! m !} s^{r+k \beta+m} \int_{0}^{\left(1+(t / s)^{c}\right)^{-1}} y^{1-\left(\frac{r+k \beta+m}{c}\right)-1}(1-y)^{\left(\frac{r+k \beta+m+c}{c}\right)-1} d y\right. \\
& +\sum_{k, m=0}^{\infty} \frac{(-1)^{k+1}((k+1) \lambda)^{m} \alpha^{k+1} \beta}{k ! m !} \frac{s^{r+k \beta+\beta+m}}{c} \int_{0}^{\left(1+(t / s)^{c}\right)^{-1}} y^{1-\left(\frac{r+k \beta+\beta+m}{c}\right)-1}(1-y)^{\left(\frac{r+k \beta+\beta+m}{c}\right)-1} d y \\
& \left.+\sum_{k, m=0}^{\infty} \frac{(-1)^{k+1}((k+1) \lambda)^{m} \alpha^{k+1} \lambda}{k ! m !} \frac{s^{r+k \beta+\beta+m+1}}{c} \int_{0}^{\left(1+(t / s)^{c}\right)^{-1}} y^{1-\left(\frac{r+k \beta+\beta+m+1}{c}\right)-1}(1-y)^{\left(\frac{r+k \beta+\beta+m+1}{c}\right)-1} d y\right] .
\end{aligned}
$$


Consequently, the $r^{\text {th }}$ conditional moment of the LLoGMW distribution is given by

$$
\begin{aligned}
E\left(X^{r} \mid X>t\right) & =\frac{1}{\bar{G}(t)}\left[\sum_{k, m=0}^{\infty} \frac{(-1)^{k+1}(k \lambda)^{m} \alpha^{k}}{k ! m !} s^{r+k \beta+m} \mathscr{B}_{\left(1+(t / s)^{c}\right)^{-1}}\left(1-\left(\frac{r+k \beta+m}{c}\right),\left(\frac{r+k \beta+m+c}{c}\right)\right)\right. \\
& +\sum_{k, m=0}^{\infty} \frac{(-1)^{k+1}((k+1) \lambda)^{m} \alpha^{k+1} \beta}{k ! m !} \frac{s^{r+k \beta+\beta+m}}{c} \mathscr{B}_{\left(1+(t / s)^{c}\right)^{-1}}\left(1-\left(\frac{r+k \beta+\beta+m}{c}\right),\left(\frac{r+k \beta+\beta+m}{c}\right)\right) \\
& \left.+\sum_{k, m=0}^{\infty} \frac{(-1)^{k+1}((k+1) \lambda)^{m} \alpha^{k+1} \lambda}{k ! m !} \frac{s^{r+k \beta+\beta+m+1}}{c} \mathscr{B}_{\left(1+(t / s)^{c}\right)^{-1}}\left(1-\left(\frac{r+k \beta+\beta+m+1}{c}\right),\left(\frac{r+k \beta+\beta+m+1}{c}\right)\right)\right],
\end{aligned}
$$

where $\mathscr{B}_{x}(a, b)=\int_{0}^{x} y^{a-1}(1-y)^{b-1} d y$ is the incomplete beta function. The mean residual lifetime function of the LLoGMW distribution is given by $E(X \mid X>t)-t$.

\section{Mean Deviations}

The amount of scatter in a population is evidently measured to some extent by the totality of deviations from the mean and median. These are known as the mean deviation about the mean and the mean deviation about the median are defined by $\delta_{1}(x)=\int_{0}^{\infty}|x-\mu| g(x) d x$ and $\delta_{2}(x)=\int_{0}^{\infty}|x-M| g(x) d x$, respectively, where $\mu=E(X)$ and $M=\operatorname{Median}(X)$. The measures $\delta_{1}(x)$ and $\delta_{2}(x)$ can be calculated using the relationships $\delta_{1}(x)=2 \mu G(\mu)-2 \mu+2 \int_{\mu}^{\infty} x g(x) d x$ and $\delta_{2}(x)=-\mu+2 \int_{M}^{\infty} x g(x) d x$, respectively. When $r=1$, we get the mean $\mu=E(X)$. Note that $T(\mu)=\int_{\mu}^{\infty} x g(x) d x$ is given by

$$
\begin{aligned}
T(\mu) & =\int_{\mu}^{\infty} x g(x) d x=\left[\sum_{k, m=0}^{\infty} \frac{(-1)^{k+1}(k \lambda)^{m} \alpha^{k}}{k ! m !} s^{1+k \beta+m} \mathscr{B}_{\left(1+(\mu / s)^{c}\right)^{-1}}\left(1-\left(\frac{1+k \beta+m}{c}\right),\left(\frac{1+k \beta+m+c}{c}\right)\right)\right. \\
& +\sum_{k, m=0}^{\infty} \frac{(-1)^{k+1}((k+1) \lambda)^{m} \alpha^{k+1} \beta}{k ! m !} \frac{s^{1+k \beta+\beta+m}}{c} \mathscr{B}_{\left(1+(\mu / s)^{c}\right)^{-1}}\left(1-\left(\frac{1+k \beta+\beta+m}{c}\right),\left(\frac{1+k \beta+\beta+m}{c}\right)\right) \\
& \left.+\sum_{k, m=0}^{\infty} \frac{(-1)^{k+1}((k+1) \lambda)^{m} \alpha^{k+1} \lambda}{k ! m !} \frac{s^{k \beta+\beta+m+2}}{c} \mathscr{B}_{\left(1+(\mu / s)^{c}\right)^{-1}}\left(1-\left(\frac{k \beta+\beta+m+2}{c}\right),\left(\frac{k \beta+\beta+m+2}{c}\right)\right)\right] .
\end{aligned}
$$

Consequently, the mean deviation about the mean and the mean deviation about the median are

$$
\delta_{1}(x)=2 \mu G(\mu)-2 \mu+2 T(\mu) \quad \text { and } \quad \delta_{2}(x)=-\mu+2 T(M)
$$

respectively.

\subsection{Bonferroni and Lorenz Curves}

Bonferroni and Lorenz curves have applications not only in economics for the study income and poverty, but also in other fields such as reliability, demography, insurance and medicine. Bonferroni and Lorenz curves for the LLoGMW distribution are given by

$$
B(p)=\frac{1}{p \mu} \int_{0}^{q} x g(x) d x=\frac{1}{p \mu}[\mu-T(q)], \quad \text { and } \quad L(p)=\frac{1}{\mu} \int_{0}^{q} x g(x) d x=\frac{1}{\mu}[\mu-T(q)],
$$

respectively, where $T(q)=\int_{q}^{\infty} x g(x) d x, q=G^{-1}(p), 0 \leq p \leq 1$.

\section{Order Statistics, Moments of Order Statistics, L-Moments and Rényi Entropy}

The concept of entropy plays a vital role in information theory. The entropy of a random variable is defined in terms of its probability distribution and can be shown to be a good measure of randomness or uncertainty. In this section, we present the distribution of the order statistic, L-moments and Rényi entropy for the LLoGMW distribution.

\subsection{Order Statistics}

Order statistics play an important role in probability and statistics. In this section, we present the distribution of the $i^{\text {th }}$ order statistic from the LLoGMW distribution. The pdf of the $i^{\text {th }}$ order statistic from the LLoGMW pdf is given by

$$
\begin{aligned}
g_{i: n}(x) & =\frac{n ! g(x)}{(i-1) !(n-i) !}[G(x)]^{i-1}[1-G(x)]^{n-i} \\
& =\frac{n ! g(x)}{(i-1) !(n-i) !} \sum_{j=0}^{n-i}(-1)^{j}\left(\begin{array}{c}
n-i \\
j
\end{array}\right)[G(x)]^{j+i-1}
\end{aligned}
$$


by using the binomial expansion $[1-G(x)]^{n-i}=\sum_{m=0}^{n-i}\left(\begin{array}{c}n-i \\ m\end{array}\right)(-1)^{m}[G(x)]^{m}$, so that

$$
\begin{aligned}
g_{i: n}(x) & =\frac{1}{\mathscr{B}(i, n-i+1)} \sum_{m=0}^{n-i}\left(\begin{array}{c}
n-i \\
m
\end{array}\right) \frac{(-1)^{m}}{m+i}(m+i)[G(x)]^{m+i-1} g(x) \\
& =\sum_{m=0}^{n-i} w_{i, m} g_{m+i}(x)
\end{aligned}
$$

where $g_{m+i}(x)$ is the pdf of the exponentiated LLoGMW distribution with parameters $s, c, \alpha, \beta, \lambda$ and $(m+i), \mathscr{B}(.$, .) is the beta function and the weights $w_{i, m}$ are given by

$$
w_{i, m}=\frac{1}{\mathscr{B}(i, n-i+1)} \frac{(-1)^{m}}{m+i}\left(\begin{array}{c}
n-i \\
m
\end{array}\right)=(-1)^{m}\left(\begin{array}{c}
m+i-1 \\
m
\end{array}\right)\left(\begin{array}{c}
n \\
m+i
\end{array}\right) .
$$

\subsection{Moments of Order Statistics}

The $t^{\text {th }}$ moment of the $i^{\text {th }}$ order statistics from the LLoGMW distribution can be derived via a result of Barakat and Abdelkader (2004) as follows:

$$
E\left(X_{i: n}^{t}\right)=t \sum_{p=n-i+1}^{n}(-1)^{p-n+i-1}\left(\begin{array}{l}
p-1 \\
n-i
\end{array}\right)\left(\begin{array}{l}
n \\
p
\end{array}\right) \int_{0}^{\infty} x^{t-1}[1-G(x)]^{p} d x
$$

Note that

$$
\int_{0}^{\infty} x^{t-1}[1-G(x)]^{p} d x=\sum_{k, m=0}^{\infty} \frac{(-1)^{k}(p \alpha)^{k}(\lambda k)^{m}}{k ! m !} \int_{0}^{\infty} x^{k \beta+m+t-1}\left[1+(x / s)^{c}\right]^{-p} d x .
$$

Let $y=\left[1+(x / s)^{c}\right]^{-1}$, then

$$
\int_{0}^{\infty} x^{k \beta+m+t-1}\left[1+(x / s)^{c}\right]^{-p} d x=\frac{s^{k \beta+m+t}}{c} \int_{0}^{1} y^{p-\frac{k \beta+m+t}{c}-1}(1-y)^{\frac{k \beta+m+t}{c}-1} d y
$$

Now,

$$
E\left(X_{i: n}^{t}\right)=t \sum_{p=n-i+1}^{n} \sum_{k, m=0}^{\infty}(-1)^{p-n+i+k} \frac{\alpha^{k}(\lambda k)^{m}}{k ! m !}\left(\begin{array}{c}
p-1 \\
n-i
\end{array}\right)\left(\begin{array}{l}
n \\
p
\end{array}\right) \frac{s^{k \beta+m+t}}{c} \mathscr{B}\left(p-\frac{k \beta+m+t}{c}, \frac{k \beta+m+t}{c}\right) .
$$

\subsection{L-Moments}

The $L$-moments (Hoskings (1990)) are expectations of some linear combinations of order statistics and they exist whenever the mean of the distribution exits, even when some higher moments may not exist. They are relatively robust to the effects of outliers and are given by

$$
\lambda_{k+1}=\frac{1}{k+1} \sum_{j=0}^{k}(-1)^{j}\left(\begin{array}{l}
k \\
j
\end{array}\right) E\left(X_{k+1-j: k+1}\right), \quad k=0,1,2, \ldots \ldots .
$$

The $L$-moments of the LLoGMW distribution can be readily obtained from equation (18). The first four $L-$ moments are given by $\lambda_{1}=E\left(X_{1: 1}\right), \lambda_{2}=\frac{1}{2} E\left(X_{2: 2}-X_{1: 2}\right), \lambda_{3}=\frac{1}{3} E\left(X_{3: 3}-2 X_{2: 3}+X_{1: 3}\right)$ and $\lambda_{4}=\frac{1}{4} E\left(X_{4: 4}-3 X_{3: 4}+3 X_{2: 4}-X_{1: 4}\right)$, respectively.

5.4 Rényi Entropy

Recall that an entropy is a measure of uncertainty or variation of a random variable. Rényi entropy (1960) is an extension of Shannon entropy. It is defined by

$$
I_{R}(v)=\frac{1}{1-v} \log \left(\int_{0}^{\infty}[g(x ; s, c, \alpha, \beta, \lambda)]^{v} d x\right), v \neq 1, v>0,
$$

and tends to Shannon entropy as $v \rightarrow 1$. Putting $[g(x ; s, c, \alpha, \beta, \lambda)]^{v}=g^{v}(x)$, we can be write

$$
\begin{aligned}
g^{v}(x) & =\sum_{k=0}^{v}\left(\begin{array}{l}
v \\
k
\end{array}\right)\left(1+\left(\frac{x}{s}\right)\right)^{k} \alpha^{k} x^{\beta k-k} e^{k \lambda x}\left(\frac{c}{s}\right)^{v-k}\left(\frac{x}{s}\right)^{(c-1)(v-k)}\left(1+\left(\frac{x}{s}\right)^{c}\right)^{-2 v} e^{-v \alpha x^{\beta} e^{\lambda x}} \\
& =\sum_{k=0}^{v} \sum_{p, m=0}^{\infty}\left(\begin{array}{l}
v \\
k
\end{array}\right) \frac{(-1)^{p} \alpha^{k}}{p ! m !}\left(\frac{c}{s}\right)^{v-k} s^{-(c-1)(v-k)}(v \alpha)^{p}(\lambda(p+k))^{m} x^{\beta k-k+(c-1)(v-k)+\beta p+m}\left(1+\left(\frac{x}{s}\right)^{c}\right)^{-2 v+k} .
\end{aligned}
$$


Considering $\int_{0}^{\infty} x^{\beta k-k+(c-1)(v-k)+\beta p+m}\left(1+\left(\frac{x}{s}\right)^{c}\right)^{-2 v+k} d x$ and letting $y=\left(1+\left(\frac{x}{s}\right)^{c}\right)^{-1}$, we have

$$
\begin{aligned}
\int_{0}^{\infty} g^{v}(x) d x & =\sum_{k=0}^{v} \sum_{p, m=0}^{\infty}\left(\begin{array}{l}
v \\
k
\end{array}\right) \frac{(-1)^{p} \alpha^{k}}{p ! m !}\left(\frac{c}{s}\right)^{v-k} s^{-(c-1)(v-k)}(v \alpha)^{p}(\lambda(p+k))^{m} \int_{0}^{\infty} x^{\beta k-k+(c-1)(v-k)+\beta p+m}\left(1+\left(\frac{x}{s}\right)^{c}\right)^{-2 v+k} d x \\
& =\sum_{k=0}^{v} \sum_{p, m=0}^{\infty}\left(\begin{array}{l}
v \\
k
\end{array}\right) \frac{(-1)^{p} \alpha^{k}}{p ! m !}\left(\frac{c}{s}\right)^{v-k} s^{-(c-1)(v-k)}(v \alpha)^{p}(\lambda(p+k))^{m} \frac{s^{\beta k-k+(c-1)(v-k)+\beta p+m+1}}{c} \\
& \times \int_{0}^{1} y^{2 v-k-1-\frac{1}{c}-\frac{1}{c}(\beta k-k+(c-1)(v-k)+\beta p+m)}(1-y)^{\frac{\beta k-k+(c-1)(v-k)+\beta p+m+1}{c}-1} d y .
\end{aligned}
$$

Consequently, Rényi entropy is given by

$$
\begin{aligned}
I_{R}(v) & =\left(\frac{1}{1-v}\right) \log \left[\sum_{k=0}^{v} \sum_{p, m=0}^{\infty}\left(\begin{array}{l}
v \\
k
\end{array}\right) \frac{(-1)^{p} \alpha^{k}}{p ! m !}\left(\frac{c}{s}\right)^{v-k}(v \alpha)^{p}(\lambda(p+k))^{m} \frac{s^{\beta k-k+\beta p+m+1}}{c}\right. \\
& \left.\times \mathscr{B}\left(2 v-k-\frac{\beta k-k+(c-1)(v-k)+\beta p+m+1}{c}, \frac{\beta k-k+(c-1)(v-k)+\beta p+m+1}{c}\right)\right],
\end{aligned}
$$

for $v \neq 1$, and $v>0$.

\section{Maximum Likelihood Estimation}

Let $X \sim \operatorname{LLoGMW}(\alpha, \beta, \lambda, s, c)$ and $\boldsymbol{\theta}=(\alpha, \beta, \lambda, s, c)^{T}$ be the parameter vector. The log-likelihood $\ell=\ell(\boldsymbol{\theta})$ for a single observation $x$ of $X$ is given by

$$
\ell(\boldsymbol{\theta})=-\alpha x^{\beta} e^{\lambda x}-2 \log \left(1+(x / s)^{c}\right)+\log \left(\left(1+\left(\frac{x}{s}\right)^{c}\right) \alpha x^{\beta-1} e^{\lambda x}(\beta+\lambda x)+\frac{c}{s}\left(\frac{x}{s}\right)^{c-1}\right) .
$$

The first derivative of the log-likelihood function with respect to $\theta$ are given by

$$
\begin{gathered}
\frac{\partial \ell}{\partial \alpha}=-x^{\beta} e^{\lambda x}+\frac{\left(1+\left(\frac{x}{s}\right)^{c}\right) x^{\beta-1} e^{\lambda x}(\beta+\lambda x)}{\left(1+\left(\frac{x}{s}\right)^{c}\right) \alpha x^{\beta-1} e^{\lambda x}(\beta+\lambda x)+\frac{c}{s}\left(\frac{x}{s}\right)^{c-1}}, \\
\frac{\partial \ell}{\partial \beta}=-\alpha e^{\lambda x} x^{\beta} \log (x)+\frac{\alpha e^{\lambda x}\left(1+\left(\frac{x}{s}\right)^{c}\right)\left(x^{\beta-1}+(\beta+\lambda x) x^{\beta-1} \log (x)\right)}{\left(1+\left(\frac{x}{s}\right)^{c}\right) \alpha x^{\beta-1} e^{\lambda x}(\beta+\lambda x)+\frac{c}{s}\left(\frac{x}{s}\right)^{c-1}}, \\
\frac{\partial \ell}{\partial \lambda}=-\alpha x^{\beta+1} e^{\lambda x}+\frac{\left(1+\left(\frac{x}{s}\right)^{c}\right) \alpha x^{\beta-1}\left(x e^{\lambda x}(\beta+\lambda x+1)\right)}{\left(1+\left(\frac{x}{s}\right)^{c}\right) \alpha x^{\beta-1} e^{\lambda x}(\beta+\lambda x)+\frac{c}{s}\left(\frac{x}{s}\right)^{c-1}}, \\
\frac{\partial \ell}{\partial s}=2\left(1+(x / s)^{c}\right)^{-1}(c / s)(x / s)^{c}-\frac{\left(c+\alpha x^{\beta} e^{\lambda x}(\beta+\lambda x)\right) c(x / s)^{c-1}}{\left(1+\left(\frac{x}{s}\right)^{c}\right) \alpha x^{\beta-1} e^{\lambda x}(\beta+\lambda x)+\frac{c}{s}\left(\frac{x}{s}\right)^{c-1}},
\end{gathered}
$$

and

$$
\frac{\partial \ell}{\partial c}=-2 \frac{(x / s)^{c}(1 / s) \log (x / s)}{\left(1+(x / s)^{c}\right)}+\frac{(1 / s)(x / s)^{c-1}\left[1+\left(1+\alpha x^{\beta-1} e^{\lambda x}(\beta+\lambda x)\right) \log (x / s)\right]}{\left(1+\left(\frac{x}{s}\right)^{c}\right) \alpha x^{\beta-1} e^{\lambda x}(\beta+\lambda x)+\frac{c}{s}\left(\frac{x}{s}\right)^{c-1}} .
$$

Given a random sample of size $n$ drawn from the LLoGMW distribution, the total log-likelihood function is given by $\ell_{n}^{*}(\boldsymbol{\theta})=\sum_{i=1}^{n} \ell_{i}(\boldsymbol{\theta})$, where $\ell_{i}(\boldsymbol{\theta}), i=1,2, \ldots ., n$ is given by equation (21). The equations obtained by setting the above partial derivatives to zero are not in closed form and the values of the parameters $s, c, \alpha, \beta, \lambda$ must be found by using iterative methods. The maximum likelihood estimates of the parameters, denoted by $\widehat{\boldsymbol{\theta}}$ is obtained by solving the nonlinear equation $\left(\frac{\partial \ell_{n}^{*}(\boldsymbol{\theta})}{\partial s}, \frac{\partial \ell_{n}^{*}(\boldsymbol{\theta})}{\partial c}, \frac{\partial \ell_{n}^{*}(\boldsymbol{\theta})}{\partial \alpha}, \frac{\partial \ell_{n}^{*}(\boldsymbol{\theta})}{\partial \beta}, \frac{\partial \ell_{n}^{*}(\boldsymbol{\theta})}{\partial \lambda}\right)^{T}=\mathbf{0}$, using a numerical method such as Newton-Raphson procedure.

Let $\widehat{\boldsymbol{\theta}}=(\hat{s}, \hat{c}, \hat{\alpha}, \hat{\beta}, \hat{\lambda})$ be the maximum likelihood estimate of $\boldsymbol{\theta}$. Under the usual regularity conditions and that the parameters are in the interior of the parameter space, but not on the boundary, we have: $\sqrt{n}(\widehat{\boldsymbol{\theta}}-\boldsymbol{\theta}) \stackrel{d}{\longrightarrow} N_{5}\left(\mathbf{0}, \mathbf{I}^{-1}(\boldsymbol{\theta})\right)$, where $\mathbf{I}(\boldsymbol{\theta})$ is the expected Fisher information matrix. The asymptotic behavior is still valid if $\mathbf{I}(\boldsymbol{\theta})$ is replaced by the observed information matrix evaluated at $\widehat{\boldsymbol{\theta}}$, that is $\mathbf{J}(\widehat{\boldsymbol{\theta}})$. The multivariate normal distribution $N_{5}\left(\mathbf{0}, \mathbf{J}^{-1}(\widehat{\boldsymbol{\theta}})\right)$ with mean vector $\mathbf{0}=(0,0,0,0,0)^{T}$, can be used to construct confidence intervals (confidence regions) for the individual model parameters and for the survival and hazard rate functions. That is, the approximate $100(1-\eta) \%$ two-sided confidence intervals for $s, c, \alpha, \beta$ and $\lambda$ are given by:

$$
\widehat{s} \pm Z_{\eta / 2} \sqrt{\mathbf{I}_{s s}^{-1}(\widehat{\boldsymbol{\theta}})}, \quad \widehat{c} \pm Z_{\eta / 2} \sqrt{\mathbf{I}_{c c}^{-1}(\widehat{\boldsymbol{\theta}})}, \quad \widehat{\alpha} \pm Z_{\eta / 2} \sqrt{\mathbf{I}_{\alpha \alpha}^{-1}(\widehat{\boldsymbol{\theta}})}, \quad \widehat{\beta} \pm Z_{\eta / 2} \sqrt{\mathbf{I}_{\beta \beta}^{-1}(\widehat{\boldsymbol{\theta}})}
$$

and $\widehat{\lambda} \pm Z_{\eta / 2} \sqrt{\mathbf{I}_{\lambda \lambda}^{-1}(\widehat{\boldsymbol{\theta}})}$, respectively, where $\left.\mathbf{I}_{s s}^{-1} \widehat{\boldsymbol{\theta}}\right), \mathbf{I}_{c c}^{-1}(\widehat{\boldsymbol{\theta}}), \mathbf{I}_{\alpha \alpha}^{-1}(\widehat{\boldsymbol{\theta}}), \mathbf{I}_{\beta \beta}^{-1}(\widehat{\boldsymbol{\theta}})$, and $\left.\mathbf{I}_{\lambda \lambda}^{-1} \widehat{\boldsymbol{\theta}}\right)$, are the diagonal elements of $\mathbf{I}_{n}^{-1}(\widehat{\boldsymbol{\theta}})=(n \mathbf{I}(\widehat{\boldsymbol{\theta}}))^{-1}$, and $Z_{\eta / 2}$ is the upper $(\eta / 2)^{t h}$ percentile of a standard normal distribution. 


\section{Simulation}

In this section, we study the performance of the LLoGMW distribution by conducting various simulations for different sample sizes and different parameter values. Equation (14) is used to generate random data from the LLoGMW distribution. The simulation study is repeated for $N=1000$ times each with sample size $n=25,50,100,200,400,800$ and parameter values $I: s=2, c=5, \alpha=4, \beta=$ $2, \lambda=6, I I: s=2, c=10, \alpha=0.5, \beta=3, \lambda=5$ and $I I I: s=0.3, c=5, \alpha=3, \beta=5, \lambda=0.1$. The following quantities were computed.

(a) Mean estimate of the MLE $\hat{\vartheta}$ of the parameter $\vartheta=s, c, \alpha, \beta, \lambda$ :

$$
\frac{1}{N} \sum_{i=1}^{N} \hat{\vartheta}
$$

(b) Average bias of the MLE $\hat{\vartheta}$ of the parameter $\vartheta=s, c, \alpha, \beta, \lambda$ :

$$
\frac{1}{N} \sum_{i=1}^{N}(\hat{\vartheta}-\vartheta) .
$$

(c) Root mean squared error (RMSE) of the MLE $\hat{\vartheta}$ of the parameter $\vartheta=s, c, \alpha, \beta, \lambda$ :

$$
\sqrt{\frac{1}{N} \sum_{i=1}^{N}(\hat{\vartheta}-\vartheta)^{2}} .
$$

Table 3 presents the Mean, Average Bias and RMSE values of the parameters $s, c, \alpha, \beta$ and $\lambda$ for different sample sizes. From the results in Table 3, we can verify that as the sample size $n$ increases, the RMSEs decay toward zero. We also observe that for all the parameter values, the biases decrease as the sample size $n$ increases. Consequently, the MLE's and their asymptotic results can be used

\begin{tabular}{|c|c|c|c|c|c|c|c|c|c|c|}
\hline \multirow[b]{2}{*}{ Parameter } & \multirow[b]{2}{*}{ Sample Size } & \multicolumn{3}{|c|}{ I } & \multicolumn{3}{|c|}{ II } & \multicolumn{3}{|c|}{ III } \\
\hline & & Mean & RMSE & Bias & Mean & RMSE & Bias & Mean & RMSE & Bias \\
\hline \multirow[t]{6}{*}{$s$} & 25 & 2.0132 & 0.5922 & 0.0132 & 2.0071 & 0.0322 & 0.0071 & 0.7576 & 7.2445 & 0.4576 \\
\hline & 50 & 2.0017 & 0.2758 & 0.0017 & 2.0080 & 0.0205 & 0.0080 & 0.3132 & 0.0444 & 0.0132 \\
\hline & 100 & 1.9987 & 0.0378 & -0.0013 & 2.0066 & 0.0178 & 0.0066 & 0.3051 & 0.0166 & 0.0051 \\
\hline & 200 & 2.0006 & 0.0116 & 0.0006 & 2.0044 & 0.0137 & 0.0044 & 0.3030 & 0.0105 & 0.0030 \\
\hline & 400 & 2.0004 & 0.0040 & 0.0004 & 2.0016 & 0.0087 & 0.0016 & 0.3019 & 0.0073 & 0.0019 \\
\hline & 800 & 2.0002 & 0.0017 & 0.0002 & 2.0003 & 0.0019 & 0.0003 & 0.3008 & 0.0049 & 0.0008 \\
\hline \multirow[t]{6}{*}{$c$} & 25 & 5.0207 & 1.0932 & 0.0207 & 10.0010 & 0.0113 & 0.0010 & 6.7837 & 14.7993 & 1.7837 \\
\hline & 50 & 4.9838 & 0.2555 & -0.0162 & 10.0014 & 0.0035 & 0.0014 & 5.6523 & 13.3994 & 0.6523 \\
\hline & 100 & 4.9884 & 0.0515 & -0.0116 & 10.0011 & 0.0029 & 0.0011 & 5.0120 & 0.4995 & 0.0120 \\
\hline & 200 & 4.9953 & 0.0197 & -0.0047 & 10.0007 & 0.0021 & 0.0007 & 5.0475 & 0.3379 & 0.0475 \\
\hline & 400 & 4.9987 & 0.0076 & -0.0013 & 10.0003 & 0.0013 & 0.0003 & 5.0213 & 0.2366 & 0.0213 \\
\hline & 800 & 4.9997 & 0.0022 & -0.0003 & 10.0001 & 0.0003 & 0.0001 & 5.0205 & 0.1699 & 0.0205 \\
\hline \multirow[t]{6}{*}{$\alpha$} & 25 & 91.2716 & 609.5621 & 87.2716 & 20.1197 & 51.5365 & 19.6197 & 10.2870 & 18.3719 & 7.2870 \\
\hline & 50 & 42.7856 & 109.0369 & 38.7856 & 13.2681 & 26.1077 & 12.7681 & 6.3709 & 12.6396 & 3.3709 \\
\hline & 100 & 25.0564 & 53.5942 & 21.0564 & 9.0259 & 18.0546 & 8.5259 & 3.7501 & 6.2312 & 0.7501 \\
\hline & 200 & 15.0558 & 30.9990 & 11.0558 & 5.9891 & 12.9717 & 5.4891 & 2.5021 & 2.8763 & -0.4979 \\
\hline & 400 & 7.6672 & 13.6054 & 3.6672 & 2.5072 & 6.4722 & 2.0072 & 2.2149 & 1.5971 & -0.7851 \\
\hline & 800 & 5.3128 & 5.0712 & 1.3128 & 1.1594 & 2.3992 & 0.6594 & 2.3046 & 1.3399 & -0.6954 \\
\hline \multirow[t]{6}{*}{$\beta$} & 25 & 2.2199 & 1.1656 & 0.2199 & 3.6349 & 2.1246 & 0.6349 & 51.9368 & 123.8157 & 46.9368 \\
\hline & 50 & 2.1101 & 0.9657 & 0.1101 & 3.4019 & 1.8562 & 0.4019 & 41.8406 & 96.4675 & 36.8406 \\
\hline & 100 & 2.1306 & 0.7547 & 0.1306 & 3.3875 & 1.5670 & 0.3875 & 30.1302 & 60.7255 & 25.1302 \\
\hline & 200 & 2.0958 & 0.5966 & 0.0958 & 3.2993 & 1.3341 & 0.2993 & 17.3133 & 35.4831 & 12.3133 \\
\hline & 400 & 2.0279 & 0.4084 & 0.0279 & 3.1173 & 0.9714 & 0.1173 & 10.5755 & 12.9039 & 5.5755 \\
\hline & 800 & 2.0107 & 0.2797 & 0.0107 & 3.0468 & 0.6915 & 0.0468 & 7.9879 & 7.5405 & 2.9879 \\
\hline \multirow[t]{6}{*}{$\lambda$} & 25 & 6.1041 & 5.1188 & 0.1041 & 4.4332 & 3.9634 & -0.5668 & 44.2329 & 104.6951 & 44.1329 \\
\hline & 50 & 6.0555 & 4.3036 & 0.0555 & 4.5254 & 3.5493 & -0.4746 & 31.4290 & 77.9436 & 31.3290 \\
\hline & 100 & 5.7192 & 3.3216 & -0.2808 & 4.4157 & 3.0721 & -0.5843 & 19.0590 & 42.0448 & 18.9590 \\
\hline & 200 & 5.7635 & 2.6715 & -0.2365 & 4.5277 & 2.6560 & -0.4723 & 8.7349 & 24.3658 & 8.6349 \\
\hline & 400 & 5.9415 & 1.8461 & -0.0585 & 4.8091 & 1.9597 & -0.1909 & 3.7954 & 7.0249 & 3.6954 \\
\hline & 800 & 5.9868 & 1.2654 & -0.0132 & 4.9308 & 1.3994 & -0.0692 & 2.1657 & 4.2199 & 2.0657 \\
\hline
\end{tabular}
for estimating and constructing confidence intervals even for reasonably small sample sizes.

Table 3. Monte Carlo Simulation Results 


\section{Estimation with Censored Samples}

In this section, we present maximum likelihood estimates of the parameters of the LLoGMW distribution under Type I right censoring scheme. An application is presented in section 9.3.

\subsection{MLE for Type I Right Censored Data}

Type I right censored data arises when the study is conducted over a specified time period that can terminate before all the units have failed. Each individual has a fixed censoring time $C_{i}$, so that the complete failure time of an individual will be known only if it is less than or equal to the censoring time $T_{i} \leq C_{i}$; otherwise, only a lower bound of the individual lifetime is available $T_{i}>C_{i}$. The data for this design are conveniently indicated by pairs of random variables $\left(T_{i}, \epsilon_{i}\right), i=1, \ldots, n$.

Let $\boldsymbol{\theta}=(\alpha, \beta, \lambda, s, c)^{T}$, then the likelihood function, $L(\boldsymbol{\theta})$, from LLoGMW distribution with pdf $g_{L L O G M W}(\cdot)$ and survival function $S_{L L o G M W}(\cdot)=1-G_{L L O G M W}(\cdot)$ can be written as

$$
L(\boldsymbol{\theta})=\prod_{i=1}^{n} g_{L L o G M W}\left(t_{i}\right)^{\epsilon_{i}} S_{L L O G M W}\left(t_{i}\right)^{1-\epsilon_{i}} .
$$

The log-likelihood function, $l(\Theta)$, based on data, from Equations (8) and (7) is

$$
\begin{aligned}
l(\boldsymbol{\theta}) & =\sum_{i=1}^{n} \epsilon_{i}\left(-\alpha t_{i}^{\beta} e^{\lambda t_{i}}-2 \log \left(1+\left(t_{i} / s\right)^{c}\right)+\log \left(\left(1+\left(\frac{t_{i}}{s}\right)^{c}\right) \alpha t_{i}^{\beta-1} e^{\lambda t_{i}}\left(\beta+\lambda t_{i}\right)+\frac{c}{s}\left(\frac{t_{i}}{s}\right)^{c-1}\right)\right) \\
& +\sum_{i=1}^{n}\left(1-\epsilon_{i}\right) \ln \left\{\left(1+\left(\frac{t_{i}}{s}\right)^{c}\right)^{-1} \exp \left(-\alpha t_{i}^{\beta} e^{\lambda t_{i}}\right)\right\} .
\end{aligned}
$$

The MLEs $\hat{\boldsymbol{\theta}}=(\hat{\alpha}, \hat{\beta}, \hat{\lambda}, \hat{s}, \hat{c})$ are obtained from the numerical maximization of log likelihood function.

\section{Applications}

To illustrate the flexibility of the LLoGMW distribution and its sub-models for data modeling, real data examples are presented. Estimates of the parameters of LLoGMW distribution with standard errors (in parentheses), Akaike Information Criterion (AIC), Bayesian Information Criterion (BIC), Cramer von Mises $\left(W^{*}\right)$, Anderson-Darling $\left(A^{*}\right)$, and sum of squares (SS) from the probability plots are displayed for each data set. The LLoGMW distribution is also compared with the gamma-Dagum (GD) (Oluyede et al. (2014)) and beta modified Weibull (BMW) (Nadarajah et al. (2011)) distributions. The GD and BMW pdfs are given by

$$
g_{G D}(x)=\frac{\lambda \beta \delta x^{-\delta-1}}{\theta^{\alpha} \Gamma(\alpha)}\left(1+\lambda x^{-\delta}\right)^{-\beta-1}\left(-\log \left[1-\left(1+\lambda x^{-\delta}\right)^{-\beta}\right]\right)^{\alpha-1}\left[1-\left(1+\lambda x^{-\delta}\right)^{-\beta}\right]^{\frac{1}{\theta}-1}, \quad x>0,
$$

and

$$
g_{B M W}(x)=\frac{\alpha x^{\gamma-1}(\gamma+\lambda x) \exp (\lambda x)}{\mathscr{B}(a, b)} e^{-b \alpha x^{\gamma} \exp (\lambda x)}\left(1-e^{-\alpha x^{\gamma} \exp (\lambda x)}\right)^{a-1}, x>0
$$

respectively.

The maximum likelihood estimates (MLEs) of the LLoGMW parameters $\boldsymbol{\theta}=(s, c, \alpha, \beta, \lambda)$ are computed by maximizing the objective function via the subroutine NLMIXED in SAS and nlm in R (2011). The estimated values of the parameters (standard error in parenthesis), -2log-likelihood statistic, Akaike Information Criterion, $A I C=2 p-2 \ln (L)$, and Bayesian Information Criterion, $B I C=p \ln (n)-$ $2 \ln (L)$, where $L=L(\widehat{\theta})$ is the value of the likelihood function evaluated at the parameter estimates, $n$ is the number of observations, and $p$ is the number of estimated parameters are presented in Tables 4 and 5 . The LLoGMW distribution is fitted to the data sets and these fits are compared to the fits using LLoGW, LLoGR, LLoGE, LLoG, W, E, R, $\operatorname{LLoGMW}(s, 1, \alpha, \beta, \lambda), \operatorname{LLoGMW}(s, 1, \alpha, 1,0)$, and $\operatorname{LLoGMW}(s, 1, \alpha, 2,0)$ distributions. The issues of existence and uniqueness of the MLEs are of theoretical interest and have been studied by several authors for different distributions including Seregin (2010), Santos Silva and Tenreyro (2010), Zhou (2009), and Xia, Mi and Zhou (2009).

The likelihood ratio (LR) test can be used to compare the fit of the LLoGMW distribution with its sub-models for a given data set. For example, to test $\beta=1$, the $\mathrm{LR}$ statistic is $\omega=2[\ln (L(\hat{\alpha}, \hat{\beta}, \hat{\lambda}, \hat{s}, \hat{c}))-\ln (L(\tilde{\alpha}, 1, \tilde{\lambda}, \tilde{s}, \tilde{c}))]$, where $\hat{\alpha}, \hat{\beta}, \hat{\lambda}, \hat{s}$, and $\hat{c}$ are the unrestricted estimates, and $\tilde{\alpha}, \tilde{\lambda}, \tilde{s}$, and $\tilde{c}$ are the restricted estimates. The LR test rejects the null hypothesis if $\omega>\chi_{\epsilon}^{2}$, where $\chi_{\epsilon}^{2}$ denote the upper $100 \epsilon \%$ point of the $\chi^{2}$ distribution with 1 degrees of freedom.

Plots of the histogram of the data together with the fitted densities, as well as probability plots (Chambers, Cleveland, Kleiner and Tukey (1983)) are presented. Probability graphs are obtained by plotting $G_{L L o G M W}\left(x_{(j)} ; \hat{s}, \hat{c}, \hat{\alpha}, \hat{\beta}, \hat{\lambda}\right)$ against $(j-0.375) /(n+0.25), j=$ $1,2, \cdots, n$, where $x_{(j)}$ are the ordered values of the observed data. The measures of closeness are given by the sum of squares (SS), where

$$
S S=\sum_{j=1}^{n}\left[G_{L L O G M W}\left(x_{(j)}\right)-\left(\frac{j-0.375}{n+0.25}\right)\right]^{2} .
$$

As described by Chen and Balakrishnan (1995), $W^{*}$ and $A^{*}$ represent the goodness-of-fit statistics. These statistics can be used to verify which distribution fits better to the data. In general, the smaller the values of $W^{*}$ and $A^{*}$, the better the fit. 


\subsection{Failure Times}

The data represent the failure times of 50 components (per 1000h), (Murthy et al. (2004)). When optimizing the maximum likelihood function, initial values for the LLoGMW model in the $\mathrm{R}$ code are set at $s=0.1, c=0.1, \alpha=0.1, \beta=0.2, \lambda=0.2$. Estimates of the parameters of LLoGMW distribution and its related sub-models, AIC, BIC, $\mathrm{W}^{*}, \mathrm{~A}^{*}$ and SS are give in Table 4 . Plots of the fitted densities and the histogram, observed probability vs predicted probability are given in Figures 3 and 4 . The estimated variancecovariance matrix for the LLoGMW distribution is given by:

$$
\left(\begin{array}{ccccc}
0.239767 & -0.019997 & 0.006535 & -0.106496 & -0.031479 \\
-0.019997 & 0.016158 & -0.001553 & -0.009204 & 0.013308 \\
0.006535 & -0.001553 & 0.000819 & -0.024398 & -0.002090 \\
-0.106496 & -0.009204 & -0.024398 & 2.152071 & -0.190631 \\
-0.031479 & 0.013308 & -0.002090 & -0.190631 & 0.051011
\end{array}\right),
$$

and the $95 \%$ confidence intervals for the model parameters are given by $s \in(1.2938 \pm 1.96 \times 0.4897), c \in(0.7758 \pm 1.96 \times 0.1271), \alpha \in$ $(0.0082 \pm 1.96 \times 0.0286), \beta \in(0.7063 \pm 1.96 \times 1.4670)$ and $\lambda \in(0.2456 \pm 1.96 \times 0.2259)$, respectively.

Table 4. Estimates of Models for Failure Time Data

\begin{tabular}{|c|c|c|c|c|c|c|c|c|c|c|c|c|}
\hline \multirow[b]{2}{*}{ Model } & \multicolumn{5}{|c|}{ Estimates } & \multicolumn{7}{|c|}{ Statistics } \\
\hline & $s$ & $c$ & $\alpha$ & $\beta$ & $\lambda$ & $-2 \log L$ & $A I C$ & $A I C C$ & $B I C$ & $W^{*}$ & $A^{*}$ & $S S$ \\
\hline LLoGMW & $\begin{array}{c}1.2938 \\
(0.4897)\end{array}$ & $\begin{array}{c}0.7758 \\
(0.1271)\end{array}$ & $\begin{array}{c}0.0082 \\
(0.0286)\end{array}$ & $\begin{array}{c}0.7063 \\
(1.4670)\end{array}$ & $\begin{array}{c}0.2456 \\
(0.2259)\end{array}$ & 197.59 & 207.59 & 208.95 & 217.15 & 0.0933 & 0.6106 & 0.0843 \\
\hline LLoGW & $\begin{array}{c}0.4897 \\
(0.1585)\end{array}$ & $\begin{array}{c}129.57 \\
(78.0147)\end{array}$ & $\begin{array}{c}0.5439 \\
(0.0995)\end{array}$ & $\begin{array}{c}0.6330 \\
(0.0736)\end{array}$ & $\begin{array}{c}0.0000 \\
-\end{array}$ & 195.52 & 203.52 & 204.89 & 211.17 & 0.1315 & 0.8097 & 0.1255 \\
\hline LLoGE & $\begin{array}{c}2.2617 \\
(1.4004)\end{array}$ & $\begin{array}{c}0.6683 \\
(0.1375)\end{array}$ & $\begin{array}{c}0.1171 \\
(0.0509)\end{array}$ & $\begin{array}{c}1.0000 \\
-\end{array}$ & $\begin{array}{c}0.0000 \\
-\end{array}$ & 203.52 & 209.52 & 210.88 & 215.25 & 0.1425 & 0.8946 & 0.1312 \\
\hline LLoG & $\begin{array}{c}1.1325 \\
(0.3162)\end{array}$ & $\begin{array}{c}0.9108 \\
(0.1033)\end{array}$ & $\begin{array}{c}0.0000 \\
-\end{array}$ & $\begin{array}{c}0.0000 \\
-\end{array}$ & $\begin{array}{c}0.0000 \\
-\end{array}$ & 210.97 & 216.97 & 218.33 & 222.71 & 0.2138 & 1.3281 & 0.1788 \\
\hline LLoGR & $\begin{array}{c}1.4222 \\
(0.5275)\end{array}$ & $\begin{array}{c}0.7210 \\
(0.1114)\end{array}$ & $\begin{array}{c}0.0089 \\
(0.0035)\end{array}$ & $\begin{array}{c}2.0000 \\
-\end{array}$ & $\begin{array}{c}0.0000 \\
-\end{array}$ & 199.02 & 205.02 & 206.39 & 210.76 & 0.0970 & 0.6464 & 0.0831 \\
\hline W & $\begin{array}{l}- \\
-\end{array}$ & $\begin{array}{l}- \\
-\end{array}$ & $\begin{array}{c}0.5412 \\
(0.0995)\end{array}$ & $\begin{array}{c}0.6613 \\
(0.0748)\end{array}$ & $\begin{array}{c}0.0000 \\
-\end{array}$ & 274.04 & 280.04 & 281.41 & 285.78 & 0.2581 & 1.6530 & 3.9656 \\
\hline $\mathrm{E}$ & $\begin{array}{l}- \\
-\end{array}$ & $\begin{array}{l}- \\
-\end{array}$ & $\begin{array}{c}0.2991 \\
(0.0423)\end{array}$ & $\begin{array}{c}1.0000 \\
-\end{array}$ & $\begin{array}{c}0.0000 \\
-\end{array}$ & 290.00 & 294.00 & 295.36 & 297.82 & 0.4295 & 2.6706 & 2.9614 \\
\hline $\mathrm{R}$ & - & $\begin{array}{l}- \\
-\end{array}$ & $\begin{array}{c}0.0353 \\
(0.0050)\end{array}$ & $\begin{array}{c}2.0000 \\
-\end{array}$ & $\begin{array}{c}0.0000 \\
-\end{array}$ & 428.51 & 432.51 & 433.88 & 436.34 & 1.1592 & 6.2391 & 2.1580 \\
\hline $\operatorname{LLoGMW}(s, 1, \alpha, \beta, \lambda)$ & $\begin{array}{c}1.0494 \\
(0.2695)\end{array}$ & $\begin{array}{c}1.0000 \\
-\end{array}$ & $\begin{array}{c}0.0023 \\
(0.0075)\end{array}$ & $\begin{array}{c}0.1486 \\
(0.9275)\end{array}$ & $\begin{array}{c}0.4171 \\
(0.3264)\end{array}$ & 201.08 & 209.08 & 210.44 & 216.72 & 0.1407 & 0.8387 & 0.2838 \\
\hline $\operatorname{LLoGMW}(s, 1, \alpha, 1,0)$ & $\begin{array}{c}1.4446 \\
(0.4942)\end{array}$ & $\begin{array}{c}1.0000 \\
-\end{array}$ & $\begin{array}{c}0.0584 \\
(0.0413)\end{array}$ & $\begin{array}{c}1.0000 \\
-\end{array}$ & $\begin{array}{c}0.0000 \\
-\end{array}$ & 208.90 & 212.90 & 214.26 & 216.72 & 0.1813 & 1.1210 & 0.3391 \\
\hline $\operatorname{LLoGMW}(s, 1, \alpha, 2,0)$ & $\begin{array}{c}1.2609 \\
(0.3500) \\
\lambda\end{array}$ & $\begin{array}{c}1.0000 \\
- \\
\beta\end{array}$ & $\begin{array}{c}0.0061 \\
(0.0030) \\
\delta\end{array}$ & $\begin{array}{c}2.0000 \\
- \\
\alpha\end{array}$ & $\begin{array}{c}0.0000 \\
- \\
\theta\end{array}$ & 204.43 & 208.43 & 209.80 & 212.26 & 0.1405 & 0.8663 & 0.3071 \\
\hline GD & $\begin{array}{c}2.4246 \\
(1.7710)\end{array}$ & $\begin{array}{c}2.4620 \\
(1.4460)\end{array}$ & $\begin{array}{c}0.2251 \\
(0.1048)\end{array}$ & $\begin{array}{c}2.2607 \\
(2.1047)\end{array}$ & $\begin{array}{c}0.0272 \\
(0.0399)\end{array}$ & 205.56 & 215.56 & 216.93 & 225.12 & 0.1700 & 1.0532 & 0.1723 \\
\hline BMW & $\begin{array}{c}a \\
272.7100 \\
(3.96 \mathrm{E}-05)\end{array}$ & $\begin{array}{c}b \\
95.421 \\
(0.0001)\end{array}$ & $\begin{array}{c}\alpha \\
1.3296 \\
(0.0146)\end{array}$ & $\begin{array}{c}\lambda \\
0.0043 \\
(0.0018)\end{array}$ & $\begin{array}{c}\gamma \\
0.0276 \\
(0.0047)\end{array}$ & 198.85 & 208.85 & 210.22 & 218.41 & 0.1060 & 0.6806 & 0.1112 \\
\hline
\end{tabular}




\section{Fitted PDF}

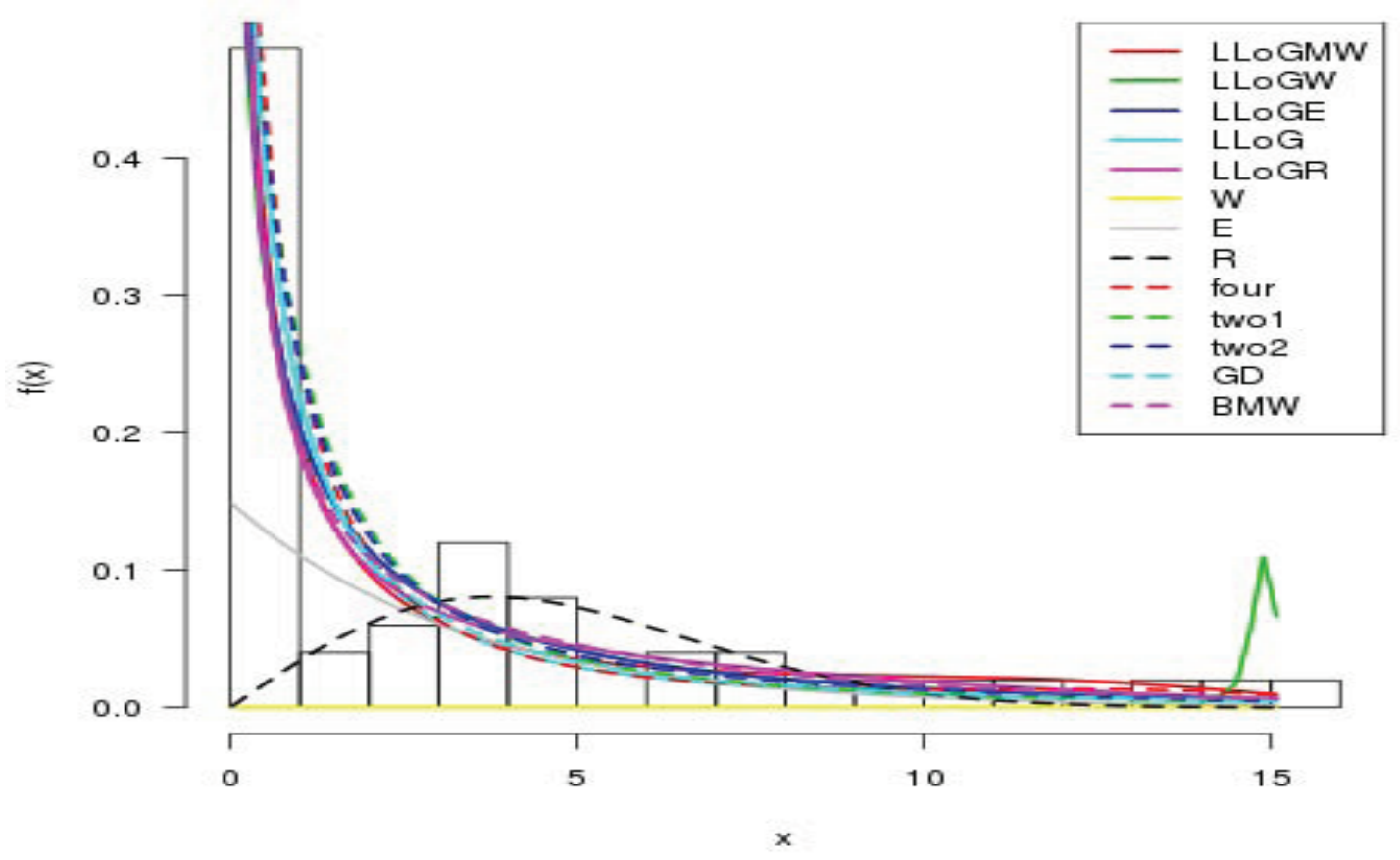

Figure 3. Fitted Densities, where Model 1 (four) is $\operatorname{LLoGMW}(s, 1, \alpha, \beta, \lambda)$; Model 2 (two1) is $\operatorname{LLoGMW}(s, 1, \alpha, 1,0)$; and Model 3 (two2) is $\operatorname{LLoGMW}(s, 1, \alpha, 2,0)$.

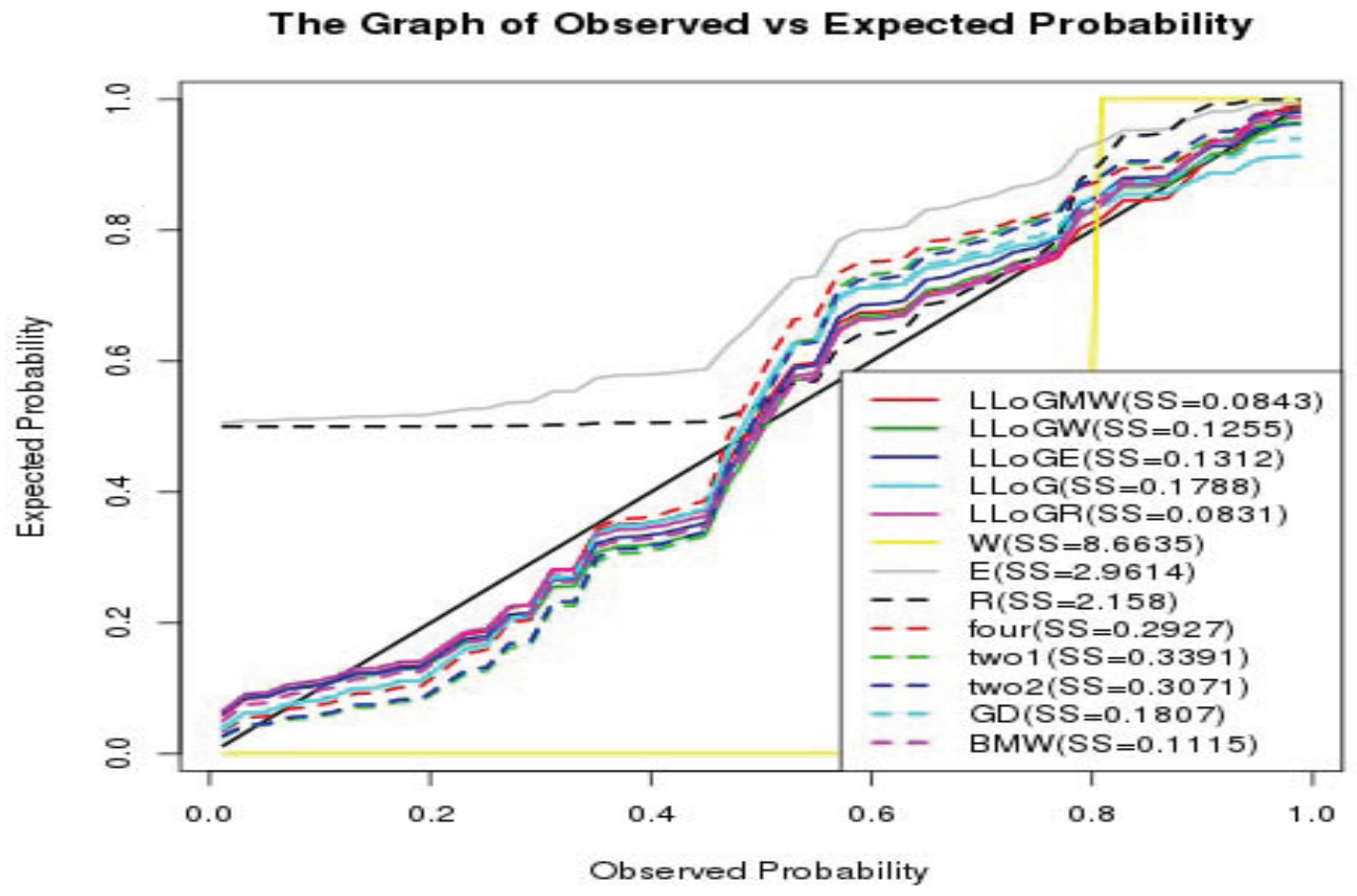

Figure 4. Probability Plots for Failure Time Data, where Model 1 (four) is $\operatorname{LLoGMW}(s, 1, \alpha, \beta, \lambda$ ); Model 2 (two1) is $\operatorname{LLoGMW}(s, 1, \alpha, 1,0)$; and Model 3 (two2) is $\operatorname{LLoGMW}(s, 1, \alpha, 2,0)$. 
Table 5. Estimates of Models for Windshield Data

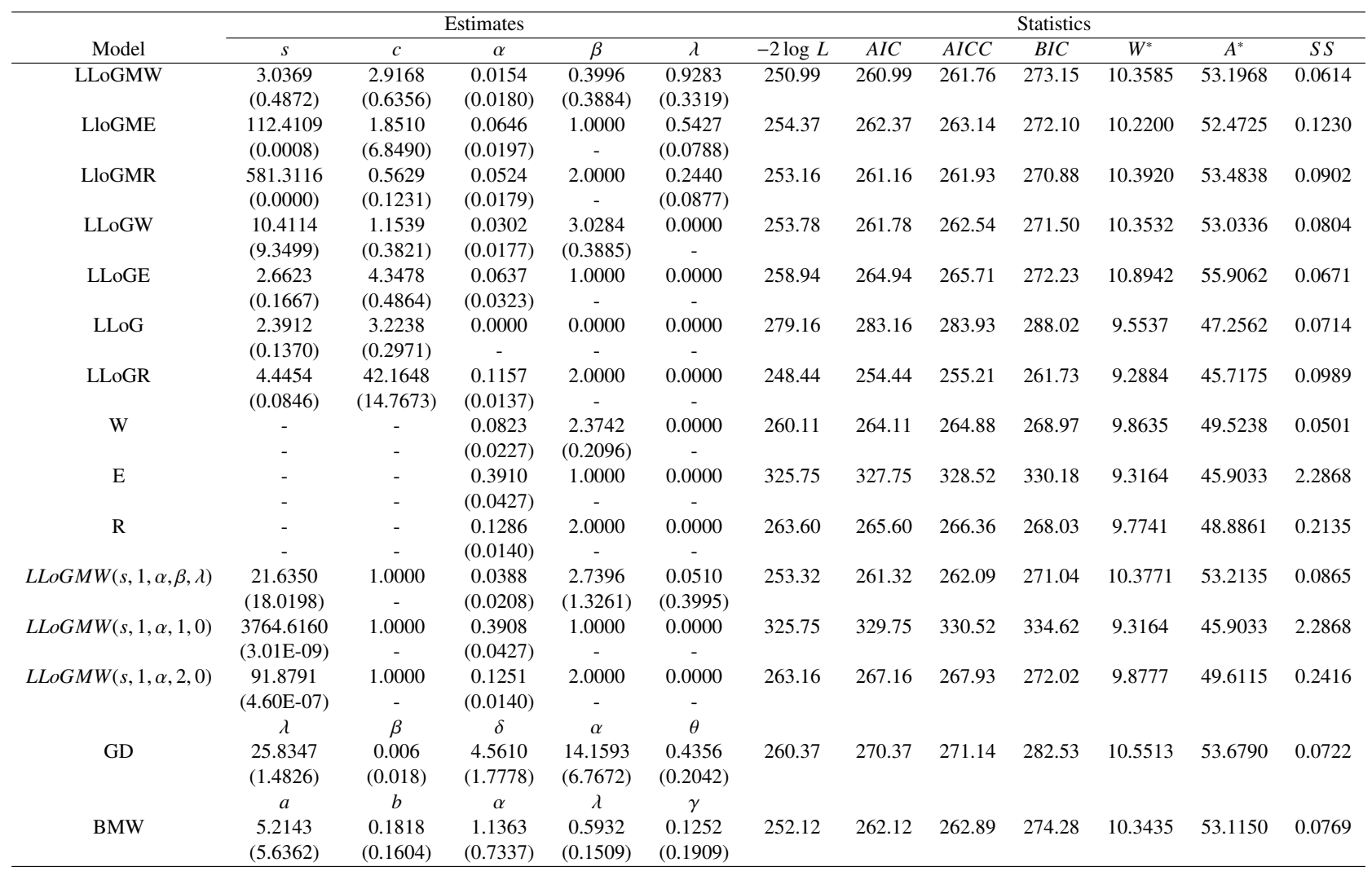

The LR test statistic for testing $H_{0}$ : LLoG against $H_{a}$ : LLoGMW and $H_{0}$ : LLoGE against $H_{a}$ : LLoGMW are 13.3803 (p-value $=$ 0.0003 ) and 5.9253 (p-value $=0.0149$ ). It is concluded that there are significant difference between LLoGMW and LLoG distributions as well between LLoGMW and LLoGE distributions. There is no significant difference between the LLoGMW and the LLoGW or LLoGR distributions based on the LR test. The values of the statistics: AIC, AICC, and BIC are smaller for the LLoGW distribution, however the goodness-of-fit statistics $W^{*}$ and $A^{*}$ are the smallest and definitely points to the LLoGMW distribution as the "best"fit for the failure time data when compared to the values for the sub-models. The goodness-of-fit statistics $W^{*}$ and $A^{*}$ are also better for the LLoGMW distribution when compared to the values for the non-nested GD and BMW distributions. Thus, there is evidence that the LLoGMW distribution is the "best" fit for the failure times data.

\subsection{Windshield Data}

This example consist of 84 Aircraft Windshield data, (Murthy et al. (2004)). When optimizing the maximum likelihood function, initial values for the LLoGMW model in the R code are set at $s=1, c=1, \alpha=1, \beta=1, \lambda=1$. The parameters estimates of the LLoGMW distribution and its related sub-models as well as the AIC, BIC, $\mathrm{W}^{*}, \mathrm{~A}^{*}$ and SS are reported in Table 5 . While Figure 5 contains the histogram and graphs of the fitted densities, Figure 6 displays observed probability against predicted probability. The estimated variance-covariance matrix for the LLoGMW distribution is given by:

$$
\left(\begin{array}{ccccc}
0.23732 & -0.06185 & 0.00567 & 0.07299 & -0.08415 \\
-0.06185 & 0.40394 & 0.00352 & 0.02730 & -0.08101 \\
0.00567 & 0.00352 & 0.00032 & 0.00325 & -0.00546 \\
0.07299 & 0.02730 & 0.00325 & 0.15084 & -0.09335 \\
-0.08415 & -0.08101 & -0.00546 & -0.09335 & 0.11014
\end{array}\right),
$$

and the $95 \%$ confidence intervals for the model parameters are given by $s \in(3.0369 \pm 1.96 \times 0.4872), c \in(2.9168 \pm 1.96 \times 0.6356), \alpha \in$ $(0.0154 \pm 1.96 \times 0.0180), \beta \in(0.3996 \pm 1.96 \times 0.3884)$ and $\lambda \in(0.9283 \pm 1.96 \times 0.3319)$, respectively. 


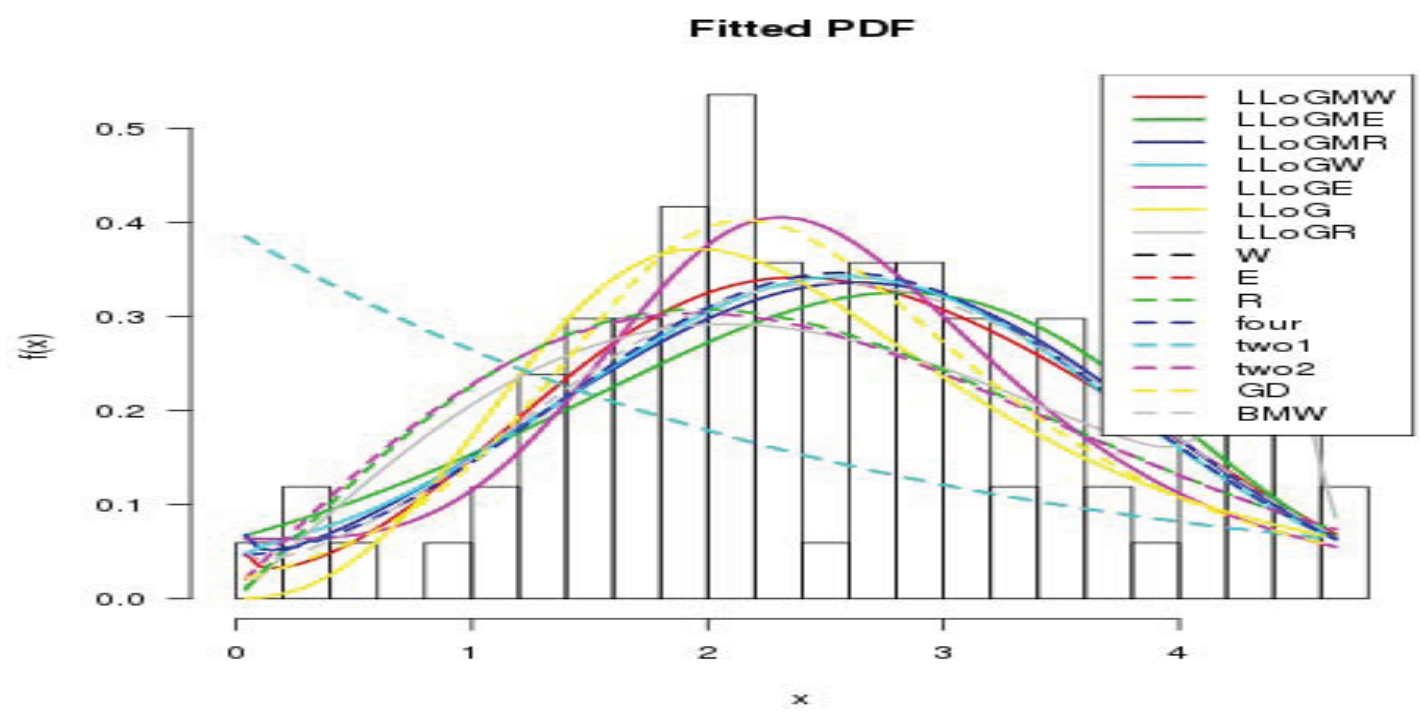

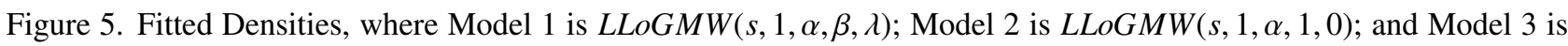
$\operatorname{LLoGMW}(s, 1, \alpha, 2,0)$.

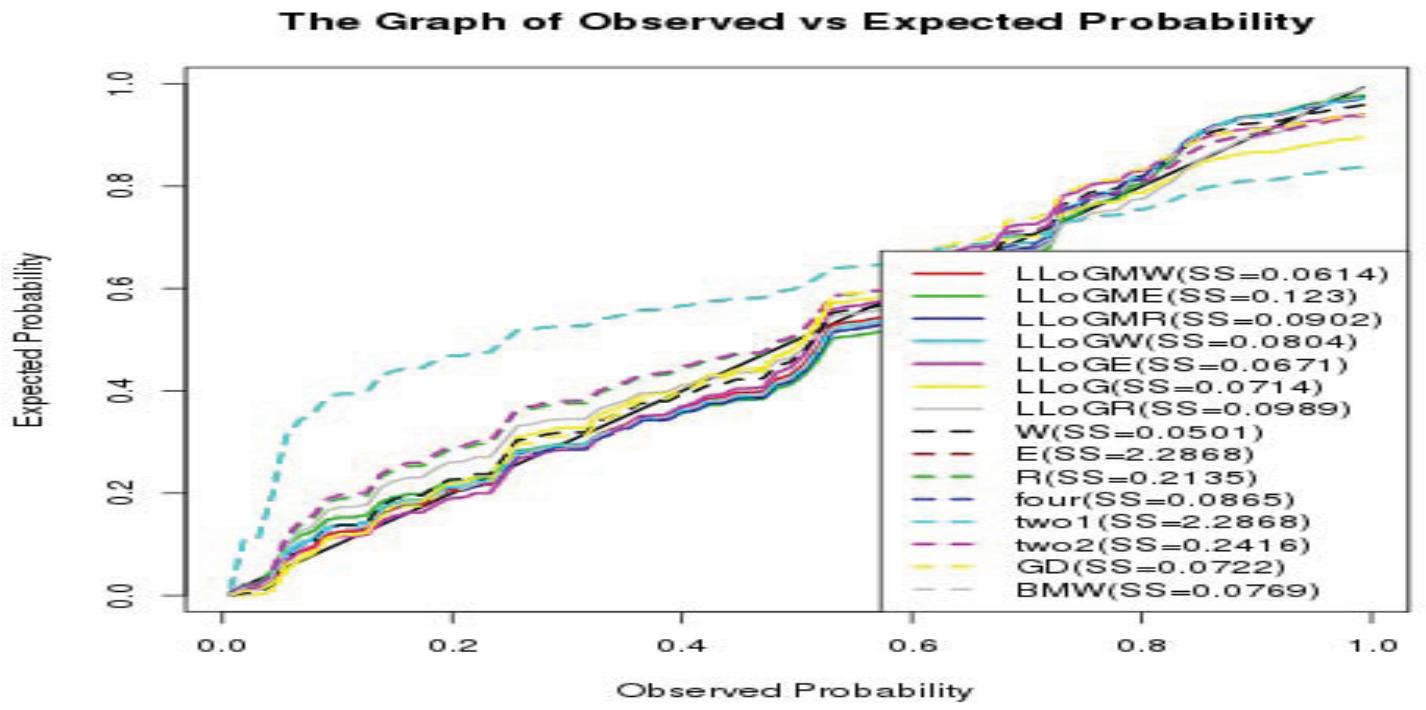

Figure 6. Probability Plots for Aircraft Windshield data, where 'four' is the four parameter model: $L L o G M W(s, 1, \alpha, \beta, \lambda)$; two1 is the model: $\operatorname{LLoGMW}(s, 1, \alpha, 1,0)$; and two2 is the model: $\operatorname{LLoGMW}(s, 1, \alpha, 2,0)$.

The LR test statistic for testing $H_{0}$ : LLoG against $H_{a}$ : LLoGMW and $H_{0}$ : LLoGE against $H_{a}$ : LLoGMW are 28.1716 (p-value $<0.0001$ ) and 7.9451 ( $\mathrm{p}$-value $=0.0048$ ). We can conclude that there are significant difference between LLoGMW and LLoG distributions as well between LLoGMW and LLoGE distributions. There is also a significant difference between Weibull and LLoGMW distributions based on the LR test statistic value of 9.12 ( $p$-value $=0.02774<0.05$ ) at the $5 \%$ level. The values of AIC, AICC and BIC as well as the goodness-of-fit statistics $W^{*}$ and $A^{*}$ shows that the LLOGMW distribution is significantly better than the non-nested GD and comparable to the BMW distributions. The submodel LLoGR distribution seem to the better fit for the data based on the goodness-of-fit statistics $W^{*}$ and $A^{*}$.

\subsection{Type I Right Censored Data: Remission Times of Cancer Patients}

This data set is remission times (in months) for 137 cancer patients, (Lee and Wang (2003)). The data is given in Table 6. Estimates of the parameters under Type I right censoring, AIC, AICC, BIC and SS are given in Table 7. The LLoGMW distribution is compared to the non-nested gamma log-logistic Weibull (GLLoGW) distribution (Foya, Oluyede, Fagbamigbe \& Makubate (2017)), the beta Weibull Poisson (BWP) distribution (Percontini, Blas \& Cordeiro (2013)) and the gamma Dagum (GD) distribution (Oluyede et al. 
Table 6. Remission Times of Cancer Patients Data

\begin{tabular}{cccccccccccc}
\hline 0.08 & 0.2 & 0.4 & 0.5 & 0.51 & 0.81 & $0.87^{*}$ & 0.9 & 1.05 & 1.19 & 1.26 & 1.35 \\
1.4 & 1.46 & 1.76 & 2.02 & 2.02 & 2.07 & 2.09 & 2.23 & 2.26 & 2.46 & 2.54 & 2.62 \\
2.64 & 2.69 & 2.69 & 2.75 & 2.83 & 2.87 & $3.02^{*}$ & 3.02 & 3.25 & 3.31 & 3.36 & 3.36 \\
3.48 & 3.52 & 3.57 & 3.64 & 3.7 & 3.82 & 3.88 & 4.18 & 4.23 & 4.26 & $4.33^{*}$ & 4.33 \\
4.34 & 4.4 & 4.5 & 4.51 & $4.65^{*}$ & $4.7^{*}$ & 4.87 & 4.98 & 5.06 & 5.09 & 5.17 & 5.32 \\
5.32 & 5.34 & 5.41 & 5.41 & 5.49 & 5.62 & 5.71 & 5.85 & 6.25 & 6.54 & 6.76 & 6.93 \\
6.94 & 6.97 & 7.09 & 7.26 & 7.28 & 7.32 & 7.39 & 7.59 & 7.62 & 7.63 & 7.66 & 7.87 \\
7.93 & 8.26 & 8.37 & 8.53 & $8.6^{*}$ & 8.65 & 8.66 & 9.02 & 9.22 & 9.47 & 9.74 & 10.06 \\
10.34 & 10.66 & 10.75 & $10.86^{*}$ & 11.25 & 11.64 & 11.79 & 11.98 & 12.02 & 12.03 & 12.07 & 12.63 \\
13.11 & 13.29 & 13.8 & 14.24 & 14.76 & 14.77 & 14.83 & 15.96 & 16.62 & 17.12 & 17.14 & 17.36 \\
18.1 & 19.13 & $19.36^{*}$ & 20.28 & 21.73 & 22.69 & 23.63 & $24.8^{*}$ & 25.74 & 25.82 & 26.31 & 32.15 \\
34.26 & 36.66 & 43.01 & 46.12 & 79.05 & & & & & & & \\
\hline
\end{tabular}

Table 7. Estimates of Models for Remission Times of Cancer Patients Data

\begin{tabular}{ccccccccccc}
\hline & \multicolumn{9}{c}{ Estimates } & \multicolumn{5}{c}{ Statistics } \\
\cline { 2 - 11 } Model & $s$ & $c$ & $\alpha$ & $\beta$ & $\lambda$ & $-2 \log L$ & $A I C$ & $A I C C$ & $B I C$ & $S S$ \\
\hline LLoGMW & 7.5494 & 1.7337 & 0.0233 & 0.7537 & 0.0137 & 835.85 & 845.85 & 846.30 & 860.45 & 0.0605 \\
& $(1.4867)$ & $(0.1951)$ & $(0.0216)$ & $(0.4204)$ & $(0.0275)$ & & & & \\
LLoGMR & 8.2670 & 1.4270 & 0.0003 & 2 & 0.0049 & 843.38 & 851.38 & 851.68 & 863.06 & 0.8183 \\
& $(0.0218)$ & $(0.1457)$ & $(0.0006)$ & - & $(0.0216)$ & & & & & \\
GLLoGW & $c$ & $\alpha$ & $\beta$ & $\delta$ & $\theta$ & & & & \\
& 0.0490 & 0.7239 & 0.6030 & 0.7243 & 0.1160 & 837.14 & 847.14 & 847.60 & 861.74 & 0.0861 \\
BWP & $(0.2863)$ & $(0.4611)$ & $(0.3346)$ & $(1.1169)$ & $(0.1340)$ & & & & & \\
& $c$ & $k$ & $\alpha$ & $\beta$ & $\theta$ & & & & \\
& 1.7844 & 0.0600 & 0.7550 & 0.5238 & 6.5441 & 836.77 & 846.77 & 847.22 & 861.37 & 0.0767 \\
GD & $(5.7104)$ & $(0.3940)$ & $(0.8203)$ & $(0.1706)$ & $(4.1910)$ & & & & & \\
& $\lambda$ & $\beta$ & $\delta$ & $\alpha$ & $\theta$ & & & & \\
& 7.3086 & 4.7985 & 0.9948 & 0.3398 & 0.2838 & 837.57 & 847.57 & 848.03 & 862.17 & 0.0731 \\
\hline
\end{tabular}

(2014)). Plots of the fitted densities and the histogram (Figure 7), hazard functions (Figure 8), survival functions (Figure 9), and observed probability vs predicted probability (Figure 10) for the remission times data are presented. 
Fitted PDF

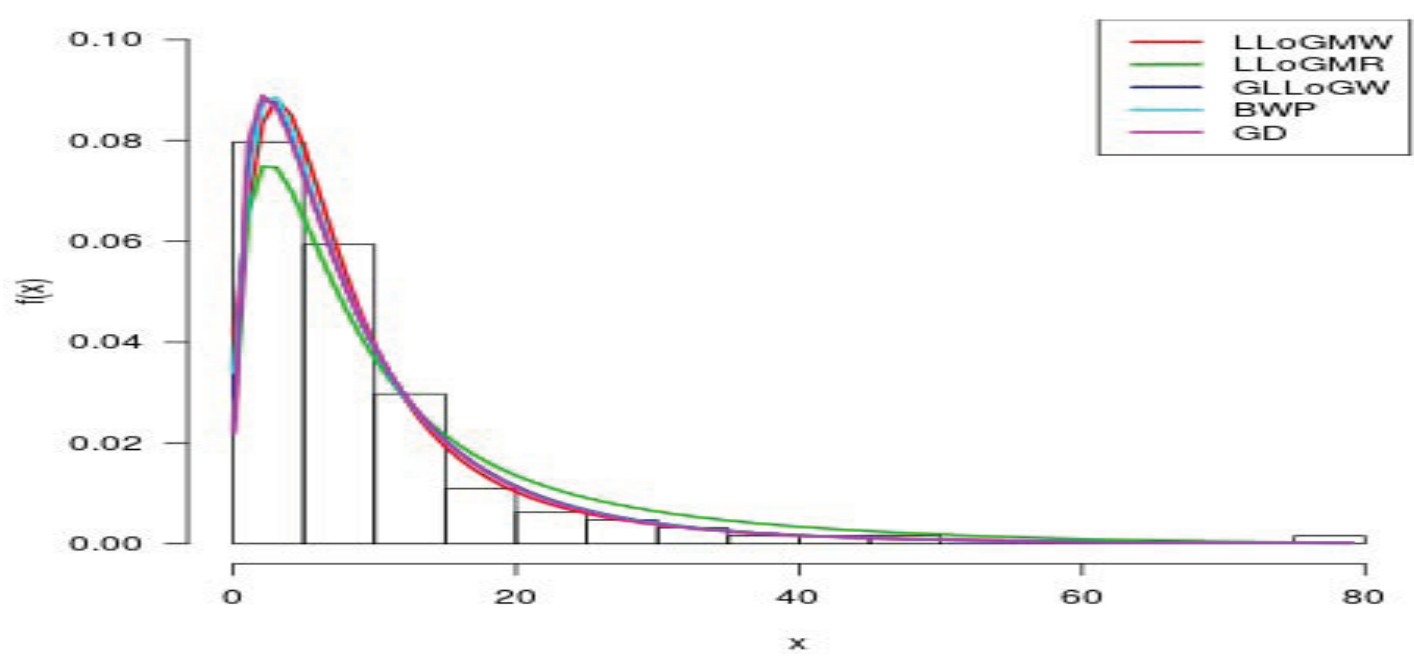

Figure 7. Graphs of fitted densities for remission times data.

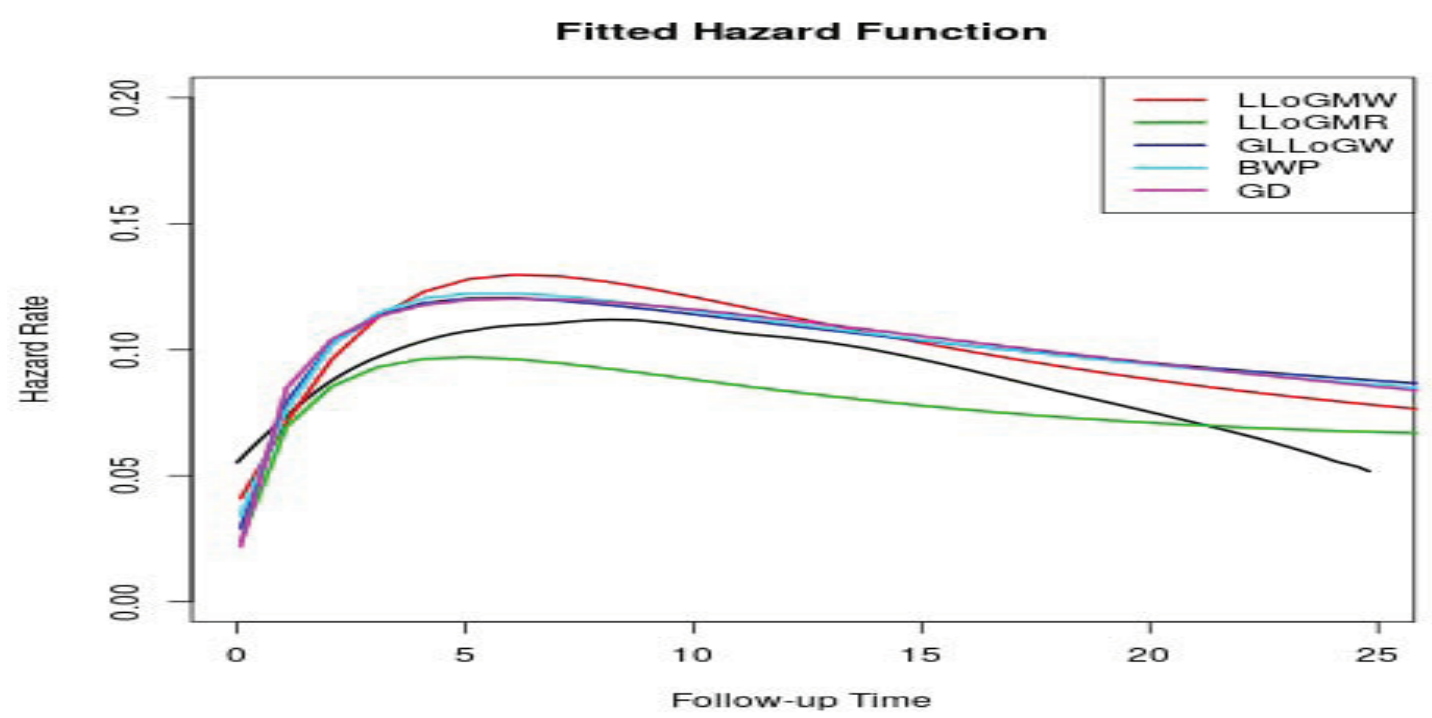

Figure 8. Graphs of estimated hazard functions for remission times data. 


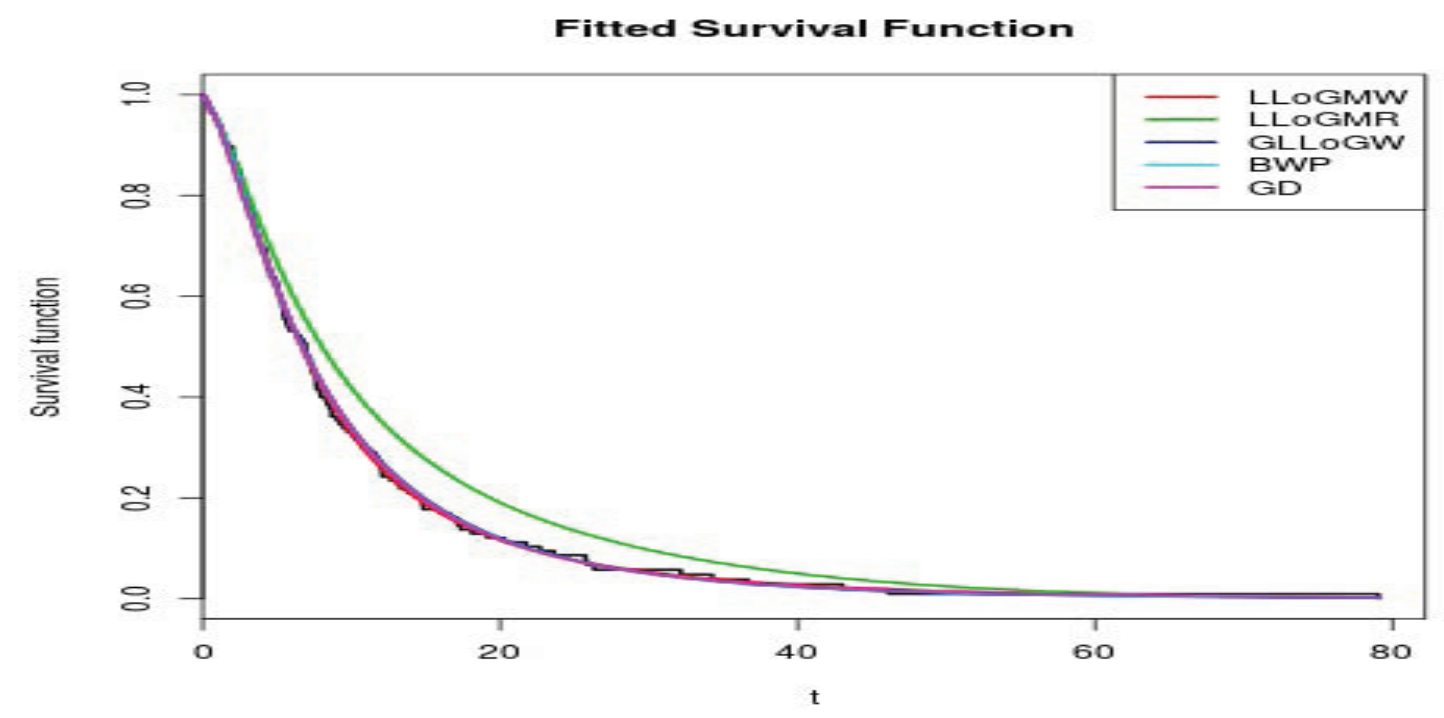

Figure 9. Graphs of estimated survival functions for remission times data.

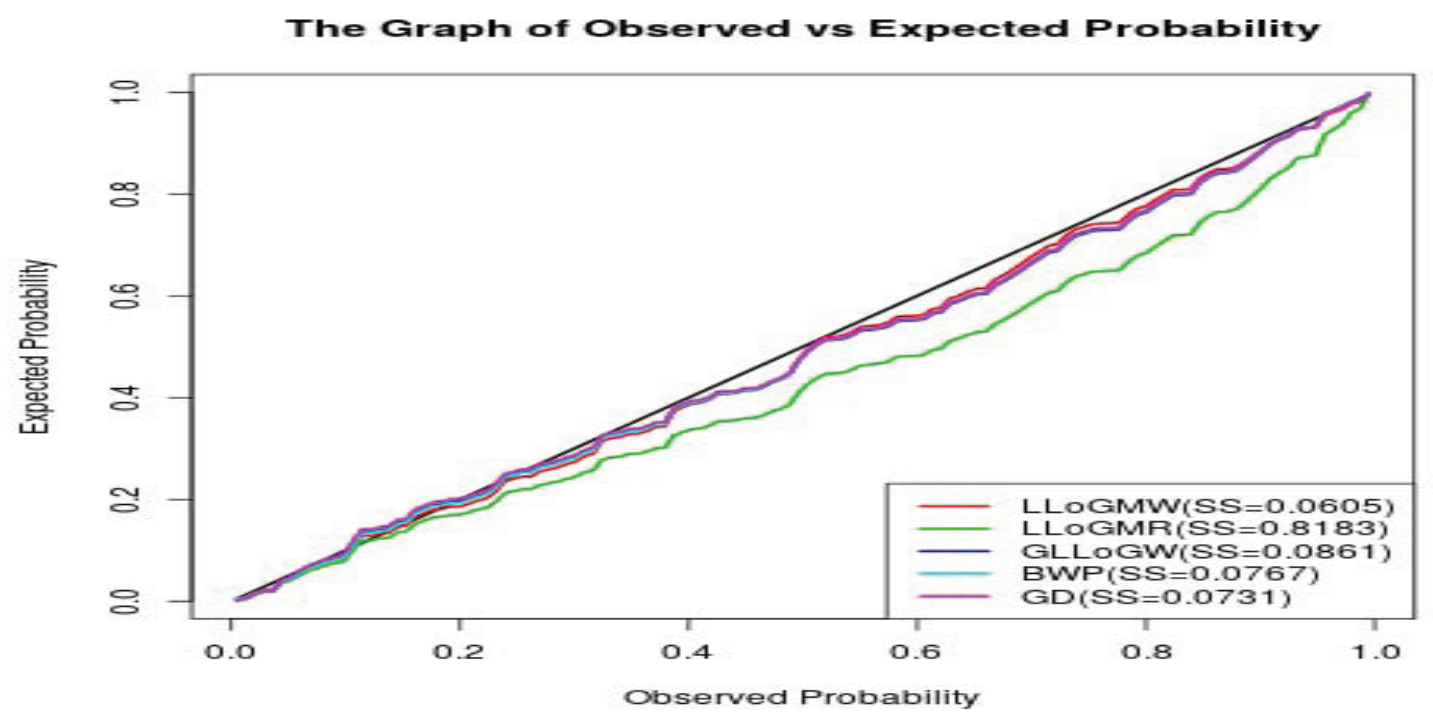

Figure 10. Probability plots for remission times data.

The LR test statistic of the hypothesis $H_{0}: L L o G M W$ against $H_{a}: L L o G M R$ is 7.54 (p-value $=0.006$ ). Also, LLoGMW distribution gives the smallest, AIC, BIC and SS values. We can conclude that LLoGMW is the best fit for remission times data under Type I right censoring scheme.

\section{Concluding Remarks}

This paper presents a new generalized distribution called the LLoGMW distribution that is shown to be suitable for applications in various areas including reliability, survival analysis, just to mention a few areas. Some of its structural properties including hazard and reverse hazard functions, quantile function, moments, conditional moments, mean deviations, Bonferroni and Lorenz curves, Rényi entropy, distribution of order statistics, maximum likelihood estimates, asymptotic confidence intervals are presented. Applications of the model to real data sets are given in order to illustrate the applicability and usefulness of the proposed distribution.

\section{Acknowledgments}

The authors are very grateful to the referees and the editor for useful comments on an earlier version of this manuscript which led to this improved version. The authors also acknowledge the support given by the staff of Department of Mathematics and Statistical Sciences at Botswana international University of Science and Technology, Palapye, BW. 


\section{References}

Barakat, H. M., \& Abdelkader, Y. H. (2004). Computing the Moments of Order Statistics from Nonidentical Random Variables. Statistical Methodology and Applications, 13, 15-26. https://doi.org/10.1007/s10260-003-0068-9

Bourguignon, M., Silva, R. B., \& Cordeiro, G. M. (2014). The Weibull-G Family of Probability Distributions. Journal of Data Science, 12, 53-68.

Burr, I. W. (1942). Cumulative Frequency Functions. Annals of Mathematical Statistics, 13, 215-232. https://doi.org/10.1214/aoms/1177731607

Burr, I. W. (1973). Parameters for a General System of Distributions to Match a grid of $\alpha_{3}$ and $\alpha_{4}$. Communications in Statistics, 2, 1-21. https://doi.org/10.1080/03610927308827052

Chambers, J., Cleveland, W., Kleiner, B., \& Tukey, P. (1983). Graphical Methods of Data Analysis, Chapman and Hall.

Chen, G., \& Balakrishnan, N. (1995). A General Purpose Approximate Goodness-of-fit Test. Journal of Quality Technology, 27, 154-161. https://doi.org/10.1080/00224065.1995.11979578

Eugene, N., Famoye, F., \& Lee, C. (2002). Beta-Normal Distribution and Its Applications. Communications in Statistics: Theory and Methods, 31, 497-512. https://doi.org/10.1081/STA-120003130

Foya, S., Oluyede, B. O., Fagbamigbe, A. F., \& Makubate, B. (2017). The Gamma Log-logistic Weibull Distribution: Model, Properties and Applcation. Electronic Journal of Applied Statistical Analysis, 10(1) , April 2017, 206-241. https://doi.org/10.1285/i20705948v10n1p206

Carrasco, M., Cordeiro, G. M., \& Ortega, E. M. (2008). A Generalized Modified Weibull Distribution for Lifetime Modeling. Computational Statistics and Data Analysis, 53(2), 450-462. https://doi.org/10.1016/j.csda.2008.08.023

Greenwood, J. A., Landwehr, J. M., Matalas, N. C., \& Willis, J. R. (1997). Probability Weighted Moments, Definitions and Relation to Parameters of Several Distributions Expressible in Inverse Form. Water Resour. Res., 15, 1049-1054.

Gupta, R. D., \& Kundu, D. (2001). Exponentiated Exponential Distribution: An Alternative to Gamma and Weibull Distributions. Biometrical Journal, 43, 117-130. https://doi.org/10.1002/1521-4036(200102)43:1;117::AID-BIMJ117;3.0.CO;2-R

Gurvich, M. R., DiBenedetto, A. T., \& Ranade, S. V. (1997). A New Statistical Distribution for Characterizing the Random Strength of Brittle Materials. Journal of Materials Science, 32, 2559-2564. https://doi.org/10.1023/A:1018594215963

Haupt, E., \& Schabe, H. (1992). A New Model for A Lifetime Distribution With Bathtub Shaped Failure Rate. Microelectronics and Reliability, 32, 633-639. https://doi.org/10.1016/0026-2714(92)90619-V

Hjorth, U. (1980). A Reliability Distribution With Increasing, Decreasing, Constant and Bathtub Failure Rates. Technometrics, 22, 99-107. https://doi.org/10.2307/1268388

Hoskings, J. R. M. (1990). L-Moments: Analysis and Estimation of Distributions Using Linear Combinations of Order Statistics. Journal of the Royal Statistical Society, B52, 105-124.

Lee, E. T., \& Wang, J., (2003). Statistical Methods for Survival Data Analysis. John Wiley \& Sons. https://doi.org/10.1002/0471458546

Lai, C. D., Xie, M., \& Murthy, D. N. P. (2003). A Modified Weibull Distribution. IEEE Transactions on Reliability, 52(1), 33-37. https://doi.org/10.1109/TR.2002.805788

Mudholkar, G. S., \& Srivastava, D. K. (1993). Exponentiated Weibull Family for Analyzing Bathtub Failure-Rate Data. IEEE Transactions on Reliability, 42, 299-302. https://doi.org/10.1109/24.229504

Murthy, D. N. P., Xie, M., \& Jiang, R. (2004). Weibull Models. Wiley Series in Probability and Statistics.

Nadarajah, S., Cordeiro, G. M., \& Ortega, E. M. M. (2011). General Results for the Beta-Modified Weibull Distribution. Journal of Statistical Computation and Simulations, 81(10), 1211-1232. https://doi.org/10.1080/00949651003796343

Nadarajah, S., \& Kotz, S. (2005). The Beta Exponential Distribution. Reliability Engineering and System Safety, 91, 689-697. https://doi.org/10.1016/j.ress.2005.05.008

Nelson, W. (1982). Lifetime Data Analysis. Wiley, New York.

Oluyede, B. O., Huang, S., \& Pararai, M. (2014). A New Class of Generalized Dagum Distribution with Applications to Income and Lifetime Data. Journal of Statistical and Econometric Methods, 3(2), 125-151.

Oluyede, B. O., Pu, S., Makubate, S., \& Qui, Y. (2018). The Gamma Weibull-G Family of Distributions. Austrian Journal of Statistics, 47, 45-76. https://doi.org/10.17713/ajs.v47i1.155

Oluyede, B. O., \& Yang, T. (2015). A New Class of Generalized Lindley Distributions with Applications. Journal of Statistical Computation and Simulations, 85(10), 2072-2100. https://doi.org/10.1080/00949655.2014.917308

Oluyede, B. O., Huang, S., \& Yang, T. (2015). A New Class of Generalized Modified Weibull Distributions with Applications. Austrian Journal of Statistics, 44, 45-68. https://doi.org/10.17713/ajs.v44i3.36 
Percontini, A., Blas, B., \& Cordeiro, G. M. (2013). The Beta Weibull Poisson Distribution. Chilean Journal of Statistics, 4(2), 3-26.

Pham, H., \& Lai, C. D. (2007). On Recent Generalizations of the Weibull Distribution. IEEE, Transaction on Reliability, $56,454-458$. https://doi.org/10.1109/TR.2007.903352

Pinho, G. B., Cordeiro, G. M., \& Nobre, J. S. (2012). The Gamma-Exponentiated Weibull Distribution. Journal of Statistical Theory and Applications, 11(4), 379-395.

Rajarshi, S., \& Rajarshi, M. B. (1988). Bathtub Distributions: A Review. Communications in Statistics-Theory and Methods, 17, 2521-2597. https://doi.org/10.1080/03610928808829761

R Development Core Team (2011). A Language and Environment for Statistical Computing, R Foundation for Statistical Computing, Vienna, Austria.

Rényi, A. (1960). On Measures of Entropy and Information. Proceedings of the Fourth Berkeley Symposium on Mathematical Statistics and Probability, 1, 547 - 561.

Santos Silva, J. M. C., \& Tenreyro, S. (2010). On the Existence of Maximum Likelihood Estimates in Poisson Regression. Econ. Lett., 107, 310-312(2010). https://doi.org/10.1016/j.econlet.2010.02.020

Seregin, A. (2010). Uniqueness of the Maximum Likelihood Estimator for k-Monotone Densities. Proc. Amer. Math. Soc. 138, 4511-4515. https://doi.org/10.1090/S0002-9939-2010-10496-3

Silva, G. O., Ortega, E. M. M., \& Cordeiro, G. M. (2010). The Beta Modified Weibull Distribution. Lifetime Data Analysis, 16, 409-430.

Xia, J., Mi, J., \& Zhou, Y. Y. (2009). On the Existence and Uniqueness of the Maximum Likelihood Estimators of Normal and Log-normal Population Parameters with Grouped Data. J. Probab. Statist., Article id 310575, 16 pages.

Zhou, C. (2009). Existence and Consistency of the Maximum Likelihood Estimator for the Extreme Index. J. Multivariate Analysis, 100, 794-815. https://doi.org/10.1016/j.jmva.2008.08.009

\section{Copyrights}

Copyright for this article is retained by the author(s), with first publication rights granted to the journal.

This is an open-access article distributed under the terms and conditions of the Creative Commons Attribution license (http://creativecommons.org/licenses/by/4.0/). 